\title{
Bundlers' dilemmas in financial markets with sampling investors
}

\author{
Milo Bianchi \\ Department of Finance, Toulouse School of Economics and TSM \\ Philippe Jehiel \\ Department of Economics, Paris School of Economics (Ecole des Ponts, Paristech) and University College \\ London
}

\begin{abstract}
We study banks' incentive to pool assets of heterogeneous quality when investors evaluate pools by extrapolating from limited sampling. Pooling assets of heterogeneous quality induces dispersion in investors' valuations without affecting their average. Prices are determined by market clearing assuming that investors can neither borrow nor short-sell. A monopolistic bank has the incentive to create heterogeneous bundles only when investors have enough money. When the number of banks is sufficiently large, oligopolistic banks choose extremely heterogeneous bundles, even when investors have little money and even if this turns out to be collectively detrimental to the banks. If, in addition, banks can originate low quality assets, even at a cost, this collective inefficiency is exacerbated and pure welfare losses arise. Robustness to the presence of rational investors and to the possibility of short-selling is discussed.
\end{abstract}

Keywords. Complex financial products, bounded rationality, disagreement, market efficiency, sampling.

JEL CLASSification. C72, D53, G14, G21.

\section{InTRODUCTION}

Many financial products such as mutual fund shares or asset-backed securities consist of claims on composite pools of assets. Pooling assets has obvious advantages, for example, in terms of improved diversification, but it may sometimes make it harder for investors to evaluate the resulting financial products. Due to time or other constraints, investors may only be able to assess limited samples of assets in the underlying pool. At the same time, as implied by many behavioral studies, investors may tend to rely too much on their own sample, trading as if it were representative of the underlying pool. ${ }^{1}$

Milo Bianchi: milo.bianchi@tse-fr.eu

Philippe Jehiel: jehiel@enpc.fr

We thank the three referees for valuable comments. We also thank Bruno Biais, Markus Brunnermeier, Xavier Gabaix, Alex Guembel, Hitoshi Matsushima, Sophie Moinas, Sébastien Pouget, Vasiliki Skreta, Dimitri Vayanos, Laura Veldkamp, and various seminar audiences for useful discussions. Bianchi acknowledges funding from the TSE Sustainable Finance Center and from ANR (Grant ANR-17-EURE-0010). Jehiel thanks the European Research Council (ERC) for funding (Grant 742816).

${ }^{1}$ This can be derived from forms of representativeness heuristic, extrapolation, overconfidence, or cursedness. We discuss these models in more details below.

(C) 2020 The Authors. Licensed under the Creative Commons Attribution-NonCommercial License 4.0. Available at https://econtheory.org. https://doi.org/10.3982/TE3726 
If investors overweight their own limited sample when evaluating pools of assets, bundling assets of heterogeneous quality may induce dispersion in investors' valuations, and this may in turn affect asset prices. We study, in such an environment, the incentives for financial institutions to design complex financial products backed by assets of heterogeneous quality. In particular, we investigate how these incentives change depending on whether potential investors have more or less money in their hands and whether there is more or less market competition in the banking system. Addressing such questions can be viewed as contributing to the large debate concerned with assessing the pros and cons of the increasing complexity of financial products. ${ }^{2}$

We develop a simple and deliberately stylized model to address our research question and later on add extra ingredients aimed at enriching some of our basic insights. We consider several banks holding assets (say, loan contracts) of different quality (say, probability of default). Banks are able to package their assets into pools as they wish and sell claims backed by these pools. We abstract from the design of possibly complex security structures and assume that banks can only sell pass-through securities (i.e., shares of the financial products). Each investor randomly samples one asset from each pool and assumes that the average value of the assets in the pool coincides with this draw considered as representative. In our stylized model, we consider an extreme version of excessive reliance on the sample and assume that no other information is used to assess the value of a pool. In particular, investors neither consider how banks may strategically allocate assets into pools ${ }^{3}$ nor do they draw any inference from market prices.

We further assume that the draws that determine the representative samples are made independently across investors. This implies that if the underlying assets of a given package are heterogeneous, the evaluations of the package are dispersed across investors. This captures the view that more complex or innovative financial products, interpreted in our framework as products backed by assets of more heterogeneous quality, are harder to evaluate. ${ }^{4}$ Hence, even starting with the same objective information, investors may end up with different assessments, which agrees with the observations made in Bernardo and Cornell (1997) and Carlin et al. (2014). ${ }^{5}$ It should be stressed that our approach does not assume a systematic bias in how the individual evaluations compare to the fundamental values. Indeed, even though the individual evaluations are

\footnotetext{
${ }^{2}$ Krugman (2007) and Soros (2009) are prominent actors of such a debate.

${ }^{3}$ Through the choice of how heterogeneous the assets are, the bank affects whether small samples are more likely to be representative of the entire pool. If banks were to pool homogenous assets, one draw would be highly representative of the assets in the pool. If banks instead tend to pool assets of heterogeneous quality (as we show they do), this is no longer the case.

${ }^{4}$ We focus on a form of complexity that comes from the pooling of heterogeneous assets, as it is typically the case for structured financial products. This form of complexity arises in particular when the distribution from which individual assets are drawn is not known to investors. Our model could be extended to investigate other forms of complexity, as we discuss in the concluding remarks.

${ }^{5}$ Mark Adelson (S\&P chief credit officer): "It [Complexity] is above the level at which the creation of the methodology can rely solely on mathematical manipulations. Despite the outward simplicity of creditratings, the inherent complexity of credit risk in many securitizations means that reasonable professionals starting with the same facts can reasonably reach different conclusions." Testimony before the Committee on Financial Services, U.S. House of Representatives, September 27, 2007. Quoted in Skreta and Veldkamp (2009).
} 
dispersed, they are correct on average due to the extrapolation from idiosyncratic samples. Despite the absence of systematic bias, market clearing prices are affected by how assets are packaged, since, as we show, prices need not be determined by the average evaluations.

To emphasize that our mechanism is unrelated to risk aversion, investors are assumed to be risk neutral. They are also wealth-constrained and cannot short-sell. Thus, pooling heterogeneous assets excludes from trading those investors who end up with low valuations, and at the same time it extracts more wealth from those investors who end up with good valuations. Prices are driven by more optimistic valuations when wealth constraints are slack, while they depend on more pessimistic valuations when wealth constraints are severe. The larger is the investors' wealth, the larger are the incentives for banks to induce disagreement by creating heterogeneous pools. As it turns out, the market structure of the banking system is also a key determinant of whether banks find it good to create heterogeneous pools. We show that more wealth and/or more competition lead to the emergence of more heterogeneous pools.

We first consider a monopolistic setting. We characterize conditions on investors' wealth under which the monopolistic bank prefers to pool all assets into a single bundle, thereby creating the largest dispersion in investors' evaluations. We also define a threshold on investors' wealth such that when investors' wealth exceeds the threshold, the bank prefers to sell its loans with some nontrivial packaging, while when wealth falls short of this threshold, disagreement decreases asset prices, so selling the loans as separate assets is optimal for the bank.

Our next central question is whether increasing competition between banks affects their incentives to pool assets of heterogeneous quality. Our main result is that these incentives are increased when several banks compete to attract investors' capital. A key observation is that, in a market with many banks, investors who happen to sample the best asset from some bundles must be indifferent between buying any of those, as otherwise the market would not clear. This implies that, irrespective of investors' wealth, the ratio between the price of a bundle and the value of its best asset must be the same across all bundles.

Each bank has then an incentive to include its most valued asset in a bundle of largest size, which can be achieved by pooling all its assets into a single bundle. We show that such a full bundling is the only equilibrium when the number of banks is sufficiently large, irrespective of investors' wealth. This should be contrasted with the monopolistic case, in which the bank has no incentive to bundle at low levels of wealth.

The main message of our paper is that more wealth in the hands of investors and/or more competition between banks to attract investors strengthen the incentives for banks to increase belief dispersion by proposing more complex financial products; that is, products backed by assets of more heterogeneous quality. In a monopolistic market with very wealthy investors, inducing belief dispersion is profitable since those who end up with less optimistic views prefer to stay out of the market. In a market with many banks, and even if investors' wealth is low, inducing belief dispersion is the best strategy, as doing otherwise would be beneficial to other banks (due to investors' comparisons of assets) and, in turn, it would attract a lower fraction of investors' wealth. 
The implications of bundling in terms of asset prices, and so in terms of banks' and investors' payoffs, are however quite different in monopoly and oligopoly. In fact, we show that even though full bundling is the only equilibrium in the highly competitive case, banks would be in some cases better off by jointly opting for a finer bundling strategy. We refer to such a situation as a bundler's dilemma. We show that bundlers' dilemmas are driven by the fact that any bank is worse off when the other banks offer larger bundles, so that bundling creates a negative externality on the other banks. When offering larger bundles, each bank is not only "stealing" investors' wealth from its competitors, but it is also decreasing the total amount of wealth attracted in the market, thereby making banks collectively worse off.

We then extend the baseline model and show that our main insights are robust when we introduce the possibility of short-selling (subject to constraints similar to those for buying) as well as a fraction of rational investors who have the correct evaluation of the various financial products. We also consider the possibility for banks to originate low quality loans, which we call lemons, at a cost that exceeds the fundamental value of the loans. We show that loan origination introduces a novel form of collective inefficiency, which has a flavor similar to that of the bundler's dilemma. In equilibrium, each bank originates a number of lemons and pools them with one high quality loan. The overall equilibrium outcome is worse for banks than what it would be if they could collectively commit to originating fewer lemons.

We also discuss some welfare implications of our results. In our baseline model, banks' strategies can distort asset prices. While in richer settings one can think of several reasons why distorted prices are not socially desirable, within our model prices induce only a transfer of wealth between banks and investors. As we assume quasi-linear preferences, those wealth transfers do not affect total welfare (defined by adding up the welfare of investors and the profits of banks). When we consider that banks can originate new loans, instead, pure welfare losses arise, and we study how those losses depend on the number of competing banks as well as on the loan origination cost.

While obviously stylized, our insights echo some evidence about the dysfunctioning of some financial markets, in particular in relation to the subprime mortgage crisis. Overly complex financial products and excessive production of low quality loans, driven by the orginate-to-distribute model, have been at the heart of the crisis (Purnanandam (2010), Allen and Carletti (2010), Maddaloni and Peydró (2011)). We believe our model sheds a novel light on this evidence by proposing an explicit mechanism through which banks would create excessive complexity and originate too many loans in an attempt to fool naive investors. Beyond the mortgage crisis, our analysis suggests several insights of independent interest that could be brought to the data. Specifically, our framework can serve as a building block for a systematic investigation of the incentives to issue assetbacked securities along the business cycle. We suggest that pool heterogeneity tends to be larger in good times, which is consistent with Downing et al. (2009) and Gorton and Metrick (2012). In terms of asset prices, existing evidence suggests that overpricing tends to be associated with low breadth of ownership (Chen et al. (2002)), higher investors' disagreement (Diether et al. (2002)), and higher asset complexity (Henderson and Pearson (2011), Célérier and Vallée (2017), and Ghent et al. (2019)). Our model 
suggests how to think in a unified way about these findings and it proposes a precise link between complexity, disagreement, and overpricing, which should be the subject of future tests.

Literature The heuristic followed by our investors builds on several closely related behavioral aspects previously discussed in the literature. Our investors extrapolate from small samples as modelled by Osborne and Rubinstein (1998). The corresponding valuation method can be related to the representativeness heuristic (in particular, to the law of small numbers) as well as to the extrapolative heuristic, which have been widely discussed in psychology as well as in the context of financial markets. ${ }^{6}$ Our formalization is most similar to Spiegler (2006) and Bianchi and Jehiel (2015), but the literature offers several other models of extrapolative investors. ${ }^{7}$

The excessive reliance on the sample used by our investors can also be related to a form of base rate neglect (they insufficiently rely on outside information such as the prior) or to a form of overconfidence (leading investors to perceive their signals as much more informative than everything else, in a similar vein as in Scheinkman and Xiong (2003)). This also exposes investors to the winner's curse, as they do not take sufficiently into account the information that other investors may have and that may be revealed by the prices. ${ }^{8}$ This is the key behavioral aspect of our model. Even starting with heterogenous beliefs, if investors were rational, they would not be willing to trade at prices above fundamentals in our setting. Several studies consider trade driven by heterogeneous beliefs in financial markets, as in Miller (1977) or Harrison and Kreps (1978). ${ }^{9}$ Compared to the previous behavioral models in financial economics, our focus on the bundling strategies of banks has no counterpart. As already highlighted, its key and novel aspect is that it structures the distribution of signals that investors receive.

A large literature on security design shows that an informed issuer may reduce adverse selection costs and promote trade by pooling its assets and create securities whose evaluations are less sensitive to private information (see, e.g., Myers and Majluf (1984), Gorton and Pennacchi (1993), DeMarzo and Duffie (1999), Biais and Mariotti (2005), DeMarzo (2005), Dang et al. (2017)). Arora et al. (2011) argue that asymmetric information can instead be exacerbated when issuers choose the content of the pools and design

\footnotetext{
${ }^{6}$ Tversky and Kahneman (1975) discuss the representativeness heuristic and Tversky and Kahneman (1971) introduce the "law of small numbers" whereby "people regard a sample randomly drawn from a population as highly representative, that is, similar to the population in all essential characteristics." In financial markets, evidence on extrapolation comes from surveys on investors' expectations (Shiller (2000), Dominitz and Manski (2011), Greenwood and Shleifer (2014)) as well as from actual investment decisions (Benartzi (2001), Greenwood and Nagel (2009), Baquero and Verbeek (2008)).

${ }^{7}$ These include De Long et al. (1990), Barberis et al. (1998), Rabin (2002), and Rabin and Vayanos (2010).

${ }^{8}$ Previous theoretical approaches to the winner's curse include the cursed equilibrium (Eyster and Rabin (2005)) or the analogy-based expectation equilibrium (Jehiel (2005) and Jehiel and Koessler (2008)) that have been applied to financial markets by Eyster and Piccione (2013), Steiner and Stewart (2015), Kondor and Koszegi (2017), or Eyster et al. (2019). See also Gul et al. (2017) for an alternative modelling of coarseness in financial markets.

${ }^{9}$ See Xiong (2013) for a recent review and Simsek (2013) for a model of financial innovation in such markets.
} 
complex securities. Part of this literature also studies how financial institutions can exploit investors' heterogeneity by offering securities catered to different investors (see, e.g., Allen and Gale (1988) for an early study and Broer (2018) and Ellis et al. (2017) for recent models). Unlike in that literature, the heterogeneity of beliefs in our setting is not a primitive of the model (in fact, we do not need any ex ante heterogeneity across investors), but it is endogenously determined by the bundling decisions of banks. Relative to security design, our focus on banks' bundling decision is complementary, and it shows that inducing dispersed valuations may be profitable even if banks cannot offer differentiated securities.

Finally, the potential benefits of bundling have been studied in several other streams of literature, from Industrial Organization (IO) to auctions. ${ }^{10}$ In particular, a recent literature on obfuscation in IO studies how firms can exploit consumers' naïveté by hiding product attributes or by hindering comparisons across products. ${ }^{11}$ Our banks can be viewed as using bundling to make it harder to evaluate their assets, but unlike in models à la Gabaix and Laibson (2006), they cannot make assets more or less visible to investors.

\section{BASELINE MODEL}

There are $N$ risk-neutral banks, indexed by $i=1, \ldots, N$. Each bank possesses a set of asset $X=\left\{X_{j}, j=1, \ldots, J\right\}$, where $x_{j} \in[0,1]$ denotes the expected payoff of asset $X_{j}$. For concreteness, $X_{j}$ may be thought of as a loan contract with face value normalized to 1 , probability of default $1-x_{j} \in[0,1]$, and zero payoff upon default. We order assets in terms of increasing expected payoff. That is, we have $x_{j} \leq x_{j+1}$ for each $j$.

Each bank may pool some of its assets and create securities backed by these pools. Each bank can package its assets into pools as it wishes. We represent the selling strategy of bank $i$ as a partition of the set of assets $X$, denoted by $\alpha^{i}=\left\{\alpha_{r}^{i}\right\}_{r}$, in which the set of bundles is indexed by $r=1,2, \ldots$. We focus on complexity considerations that arise merely from banks' bundling strategies. That is, we do not consider the use of possibly complex contracts that would map the value of the underlying pool to the payoff of the securities, and we assume that each bank $i$ simply creates pass-through securities backed by the pool $\alpha_{r}^{i}$ for each $r$. Accordingly, an investor who buys a fraction $\omega$ of the securities backed by $\alpha_{r}^{i}$ is entitled to a fraction $\omega$ of the payoffs generated by all the assets in $\alpha_{r}^{i}$. The expected payoff of bank $i$ choosing $\alpha^{i}$ is defined as

$$
\pi^{i}=\sum_{r}\left|\alpha_{r}^{i}\right| p\left(\alpha_{r}^{i}\right)
$$

where $\left|\alpha_{r}^{i}\right|$ is the number of assets contained in $\alpha_{r}^{i}$ and $p\left(\alpha_{r}^{i}\right)$ is the price of the security backed by $\alpha_{r}^{i}$. We denote the set of bundles sold by all banks by $A=\left\{\left\{\alpha_{r}^{i}\right\}_{r}\right\}_{i=1}^{N}$.

\footnotetext{
${ }^{10}$ In the context of a monopolist producing multiple goods, see, e.g., Adams and Yellen (1976) and McAfee et al. (1989). For models of auctions, see, e.g., Palfrey (1983) and Jehiel et al. (2007).

${ }^{11}$ See Spiegler (2016) for a recent review of these models and see Carlin (2009) for an application of obfuscation to financial products.
} 
There is a continuum of risk-neutral investors. ${ }^{12}$ For each bundle $\alpha_{r}^{i}$, an investor samples one basic asset from $\alpha_{r}^{i}$ at random (uniformly over all assets in $\alpha_{r}^{i}$ ) and assumes that the average expected value of the assets in $\alpha_{r}^{i}$ coincides with this draw. We assume that the draws are independent across investors. ${ }^{13}$ It follows that if $\left|\alpha_{r}^{i}\right|=1$, investors share the same correct assessment of bundle $\alpha_{r}^{i}$. But if $\left|\alpha_{r}^{i}\right|>1$, investors may attach different values to $\alpha_{r}^{i}$, depending on their draws. As already mentioned, however, bundling heterogenous assets induces only belief dispersion and no systematic bias in the average valuation across investors.

As investors are risk neutral and they buy claims on the total payoff generated by bundle $\alpha_{r}^{i}$, they care about the average expected value of the assets in $\alpha_{r}^{i}$.

Prices are determined by market clearing, assuming that investors have aggregate wealth $W$ and that they cannot borrow or short-sell (an assumption we relax in Section 5). The supply and demand of the securities backed by $\alpha_{r}^{i}$ are defined as follows. If $\alpha_{r}^{i}$ consists of $\left|\alpha_{r}^{i}\right|$ assets, the supply of $\alpha_{r}^{i}$ is

$$
S\left(\alpha_{r}^{i}\right)=\left|\alpha_{r}^{i}\right|
$$

The demand for $\alpha_{r}^{i}$ depends on the profile of valuations across all investors and all bundles. The set of these valuations can be represented as $\left[x_{1}, x_{J}\right]^{A}$, associating to each bundle $\alpha_{r}^{i} \in A$, for $r$ and $i$, a valuation $\widetilde{x}_{r, k}^{i}$ (uniformly drawn from $\alpha_{r}^{i}$ ). By the law of large numbers, each asset in each generic bundle $\alpha_{r}^{i}$ is sampled by a fraction $1 /\left|\alpha_{r}^{i}\right|$ of investors. Hence, the fraction of investors characterized by a valuation profile $\widetilde{x}_{k}=\left(\widetilde{x}_{r, k}^{i}\right)_{r, i}$ is

$$
\eta_{k}=\prod_{i} \prod_{r} \frac{1}{\left|\alpha_{r}^{i}\right|} \quad \text { for all } k
$$

The demand for $\alpha_{r}^{i}$ is defined by

$$
D\left(\alpha_{r}^{i}\right)=\frac{W}{p\left(\alpha_{r}^{i}\right)} \sum_{k} \eta_{k} \lambda_{k}\left(\alpha_{r}^{i}\right),
$$

where $\lambda_{k}\left(\alpha_{r}^{i}\right) \in[0,1]$ is the fraction of the budget of investors with valuations $\tilde{x}_{k}$ allocated to bundle $\alpha_{r}^{i}$. Given the risk-neutrality assumption, each investor allocates his entire budget to the securities perceived as most profitable. That is, denote by $\widetilde{x}_{k}\left(\alpha_{r}^{i}\right)=\widetilde{x}_{r, k}^{i}$ the valuation of bundle $\alpha_{r}^{i}$ according to the profile $\tilde{x}_{k}$. We have

$$
\lambda_{k}(\hat{\alpha})>0 \quad \text { iff } \quad \hat{\alpha} \in \underset{\alpha_{r}^{i} \in A}{\arg \max } \frac{\widetilde{x}_{k}\left(\alpha_{r}^{i}\right)}{p\left(\alpha_{r}^{i}\right)} \quad \text { and } \quad \tilde{x}_{k}(\hat{\alpha})-p(\hat{\alpha}) \geq 0
$$

and

$$
\Sigma_{\alpha_{r}^{i}} \lambda_{k}\left(\alpha_{r}^{i}\right)=1 \text { if } \max _{\alpha_{r}^{i} \in A}\left(\widetilde{x}_{k}\left(\alpha_{r}^{i}\right)-p\left(\alpha_{r}^{i}\right)\right)>0
$$

\footnotetext{
${ }^{12}$ Considering such a limiting case simplifies our analysis as it removes the randomness of prices (which would otherwise vary stochastically as a function of the profile of realizations of the assessments of the various investors).

${ }^{13}$ More generally, the insights developed below would carry over as long as there is no perfect correlation of the draws across investors.
} 
The timing is as follows. Banks simultaneously decide their selling strategies so as to maximize the expected payoff as described in (1); investors assess the value of each security according to the above-described procedure and form their demand as in (3); a competitive equilibrium emerges, which determines the price for each security so as to clear the markets for all securities.

\section{MonOPOLY}

We start by analyzing a monopolistic setting with $N=1$ (we omit the superscript $i$ for convenience), and we study the effect of investors' wealth on the incentives for the bank to bundle its assets. Intuitively, the larger is the wealth, the more optimistic are the investors who fix the market clearing price, and so the bigger the incentive for the bank to create heterogeneous bundles.

We note that bundling is profitable to the extent that only the investors who overvalue the bundle (as compared with the fundamental value) are willing to buy. The question is whether the wealth possessed by those investors is sufficient to satisfy the corresponding market clearing conditions at such high prices. An immediate observation is that bundling cannot be profitable if the aggregate wealth $W$ falls short of the fundamental value of the assets that are sold in the market, since selling assets separately exhausts the entire wealth and the payoff from any bundling cannot exceed $W$ (while it can sometimes fall short of $W$ due to the possibly pessimistic assessment of the bundle).

Another simple observation is that when investors are very wealthy $\left(W / J>J x_{J}\right.$, where $x_{J}$ is the best asset), the price of any bundle is determined by the most optimistic evaluation of the bundle-that is, by the maximum of the draws across investorsirrespective of the bank's bundling strategy. In this case, it is optimal for the bank to create as much disagreement as possible, so full bundling strictly dominates any other strategy.

More generally, the larger the aggregate wealth $W$, the more profitable it is to create bundles with several assets of heterogeneous value. While full bundling is optimal when $W$ is large enough, some nontrivial but partial bundling is optimal at intermediate levels of wealth, whereas at sufficiently low levels of wealth, the bank finds it optimal to sell its assets separately. More precisely, if wealth is so low that pooling $\left\{X_{1}, X_{2}\right\}$ and offering the other assets separately is dominated by offering all assets separately, then no other bundling can be profitable, which in turn yields the following proposition.

Proposition 1. Some bundling strictly dominates full separation if and only if $W>$ $\max \left(2\left(x_{2}+x_{1}\right), \sum_{j} x_{j}\right)$.

\section{Oligopoly}

We now consider multiple banks and observe that the incentives to offer assets in bundles are larger in markets with sufficiently many banks. As it turns out, when $N$ is large, full bundling is the only equilibrium, even at levels of wealth at which a monopolistic 
bank would sell its assets separately. We then show that bundling creates a negative externality on the other banks, which can lead banks to situations similar to a prisoner's dilemma.

\subsection{Full bundling is the only equilibrium}

Consider some partition of assets across banks. Let $\alpha_{r}$ be a generic bundle (the identity of the selling bank is not important), let $J_{r} \geq 1$ be the number of elements in $\alpha_{r}$, let $x_{r}^{*}$ be the highest value of the assets in bundle $\alpha_{r}$, let $p_{r}$ be the market clearing price of a security backed by $\alpha_{r}$, and define ${ }^{14}$

$$
\mu_{r} \equiv \frac{p_{r}}{x_{r}^{*}}
$$

We first show that when $N$ is large, market clearing requires that the ratio $\mu_{r}$ is constant across all bundles sold by all banks.

Lemma 1. There exist $\mu_{0} \in(0,1]$ and $N_{0}$ such that if $N \geq N_{0}$, then market clearing requires

$$
p_{r}=\mu_{0} x_{r}^{*} \text { for all } \alpha_{r} \in A .
$$

Moreover, $N_{0}$ can be chosen irrespective of the partition of assets into bundles.

Notice that $1 / \mu_{r}$ defines the highest returns of bundle $r$. That is, the returns perceived by those investors who happen to sample the best asset $x_{r}^{*}$ in that bundle. According to Lemma 1, market clearing requires that those highest returns should be equalized across bundles when $N$ is large enough. To have an intuition for this, notice that if a bundle $r_{1}$ had a strictly larger ratio than all other bundles, it would attract at most those investors who sample no best asset from any of the other bundles. When $N$ is large, and so the number of bundles is large, the probability of sampling no best asset from all other bundles is small. In this case, the fraction of wealth attracted by bundle $r_{1}$, and so its price, is also small, and for $N$ large enough that would contradict the premise that $r_{1}$ had a strictly higher ratio. The proof extends this intuition, showing that the markets would not clear unless the ratios $\mu_{r}$ are equated across the various bundles. That $N_{0}$ can be set independently of the partitions of assets into bundles follows because there are only finitely many possible partitions of the assets for any bank.

Lemma 1 implies that when $N$ is large, the price of each bundle is driven by its highest valued asset. This suggests that each bank has an incentive to maximize the most valued asset in a bundle, which can be achieved by pooling all assets into a single bundle. Of course, this loose intuition does not take into account that the constant of proportionality $\mu_{0}$ depends itself on the bundling strategies of the banks. But as it turns out, full bundling is the only equilibrium when $N$ is large given that, in this case, a single bank cannot have much of an effect on $\mu_{0}$.

\footnotetext{
${ }^{14}$ Notice that $\mu_{r}$ cannot be defined when $x_{r}^{*}=0$, which occurs when $x_{1}=0$ and $x_{1}$ is sold as a separate asset. In this case, its price cannot be different from 0 . Since this case is immaterial for our equilibrium construction, we ignore it in the next lemma.
} 
Proposition 2. Suppose $x_{J}>x_{J-1}$. Irrespective of $W$, there exists $N^{*}$ such that if $N \geq$ $N^{*}$, then full bundling is the only equilibrium.

To have a finer intuition as to why full bundling is an equilibrium, suppose all banks propose the full bundle and bank $j$ deviates to another bundling strategy. From Lemma 1, the fraction of wealth allocated to each bundle depends on the value of its best asset. Full bundling gives a price proportional to $x_{J}$ for all assets, while the deviating bank would at best sell $J-1$ assets at a price proportional to $x_{J}$ and one asset at a price proportional to its second best asset $x_{J-1}$. Relative to the other banks, the deviating bank would experience a loss at least proportional to $\left(x_{J}-x_{J-1}\right)$, and this remains positive irrespective of $N$. At the same time, all banks could benefit from the deviation if the total amount of wealth invested were to increase. Such an increase is bounded by the fraction of wealth not invested before the deviation, which corresponds at most to the mass of those investors who sample no best asset from any of the bundles. When $N$ is large, these investors are not many and so the increase in wealth is small, which makes the deviation not profitable.

The proposition also rules out any other possibly asymmetric equilibrium. Starting from an arbitrary profile of (possibly asymmetric) bundles, we show that the bank receiving the lowest payoff would be better off by deviating to full bundling.

\subsection{The bundler's dilemma}

Another implication of Lemma 1 is that each bank is better off when the other banks choose finer partitions than when they offer coarser partitions of their assets. Let us introduce the following definition.

Definition 1. Consider two partitions $\tilde{\alpha}^{i}$ and $\alpha^{i}$ of $X^{i}$. We say that $\tilde{\alpha}^{i}$ is coarser than $\alpha^{i}$ (or, equivalently, that $\alpha^{i}$ is finer than $\tilde{\alpha}^{i}$ ) if $\tilde{\alpha}^{i}$ can be obtained from the union of some elements of $\alpha^{i}$.

We can show that, irrespective of its strategy, each bank receives lower payoffs when the other banks offer coarser partitions than when they offer finer partitions. When the other banks offer coarser partitions, the total amount of wealth invested is lower, since the probability of sampling an asset whose value is lower than the price from all bundles is larger. At the same time, from Lemma 1, banks offering coarser partitions receive a larger fraction of this wealth, as some of their best assets would be included in larger bundles. We then have the following proposition.

Proposition 3. Consider partitions $\tilde{\alpha}$ and $\alpha$, where $\tilde{\alpha}$ is coarser than $\alpha$. If $N \geq N_{0}$, irrespective of its strategy and of $W$, each bank is better off when all other banks offer partition $\alpha$ than when they offer partition $\tilde{\alpha}$.

Proposition 3 implies in particular that each bank is better off when the other banks sell their assets separately than when they offer them in bundles. In this sense, we say that bundling creates a negative externality on the other banks. 
This externality leads to a new phenomenon, which we call bundler's dilemma (with obvious reference to the classic prisoner's dilemma). ${ }^{15}$ Full bundling can be the only equilibrium and at the same time be collectively bad for banks, in the sense that if banks could make a joint decision, they would rather choose a finer bundling strategy.

Definition 2. We have a bundler's dilemma when (i) full bundling is the only equilibrium and (ii) banks would be better off by jointly choosing a finer bundling strategy.

A special (extreme) case of the bundler's dilemma arises when banks would be collectively better off by selling their assets separately, while in equilibrium they are induced to offer the full bundle. This occurs under the following conditions.

Corollary 1. Suppose $N \geq N^{*}$ and

$$
\frac{W}{N} \in\left(J x_{1}, \frac{\sum_{j} x_{j}}{1-\left(\frac{1}{J}\right)^{N}}\right) .
$$

We have a bundler's dilemma in which full bundling is the only equilibrium while banks would collectively prefer full separation.

Equation (5) follows from simple algebra. When $W / N>J x_{1}$, the price of each bundle is strictly greater than $x_{1}$. Otherwise, all investors would be willing to buy irrespective of their draw, all wealth would be extracted, and the price of each bundle would exceed $x_{1}$, leading to a contradiction. It follows that investors who draw $X_{1}$ from all bundles, that is a fraction $(1 / J)^{N}$ of investors, do not participate and each bundle gets at most

$$
\frac{W}{N}\left(1-\left(\frac{1}{J}\right)^{N}\right)
$$

The upper bound in (5) is derived by imposing that (6) does not exceed $\sum_{j} x_{j}$ so that each bank would be better off if all assets were sold separately (in which case they earn $\left.\min \left(\frac{W}{N}, \sum_{j} x_{j}\right)\right)$. Note that if one thinks of the ratio $W / N$ as remaining constant as $N$ varies, the corollary implies that a bundler's dilemma arises if the ratio $W / N$ lies in $\left(J x_{1}, \sum_{j} x_{j}\right)$ and $N$ lies above the threshold $N^{*}$.

\subsection{The bundler's dilemma in a cherry/lemons market}

Corollary 1 describes an extreme form of bundler's dilemma in which prices fall short of fundamentals. We now show that the range over which such a dilemma can occur is considerably larger, in particular covering cases in which the prices are above the fundamentals. To illustrate this most simply, we specialize the set of asset as

$$
X=\left\{x_{j}=0 \text { for } j \leq J-1 \text {, and } x_{J}=x>0\right\} .
$$

\footnotetext{
${ }^{15}$ We thank Laura Veldkamp for suggesting this terminology.
} 
That is, each bank has one good asset (a cherry) with value $x$ and $J-1$ assets with value 0 , which we call lemons. While stylized, this setting allows us to capture most clearly the possibility of pooling high and low quality assets. It also simplifies considerably the bundling strategy, which amounts to deciding the number of lemons included in the pool together with the cherry. This simplification enables an explicit characterization of necessary and sufficient conditions for having full bundling in equilibrium as well as when bundlers' dilemmas arise.

If $N$ banks choose a symmetric strategy and offer a pool of size $J$, the payoff for each bank is

$$
\pi(J)=\min \left(J x,\left(1-\left(\frac{J-1}{J}\right)^{N}\right) \frac{W}{N}\right)
$$

Define

$$
\hat{N}(W)=\max \left\{N: J x \leq\left(1-\left(\frac{J-1}{J}\right)^{N}\right) \frac{W}{N}\right\} .
$$

Whenever $N$ is smaller than $\hat{N}(W)$, if all banks bundle all their assets, the resulting market clearing price of each bundle is $J x$, which is clearly the most a bank can hope to get. It follows that when $N \leq \hat{N}(W)$, full bundling is an equilibrium, and it can be shown that it is the only equilibrium. More interesting is the existence of $\bar{N}(W)$ such that if $N \geq \bar{N}(W)$, full bundling is the only equilibrium. The threshold $\bar{N}(W)$ is determined so that when all banks offer the full bundle, it is not profitable to deviate and attract all those who sample a lemon from the other banks. The threshold $\bar{N}(W)$ decreases in $W$ and $\bar{N}(W) \rightarrow N^{*}$ when $W \rightarrow 0$, where

$$
N^{*}=\min \left\{N:\left(\frac{J-1}{J}\right)^{N-1} \leq\left(1-\left(\frac{J-1}{J}\right)^{N}\right) \frac{1}{N}\right\} .
$$

The threshold $N^{*}$ ensures that, as in Proposition 2 for the baseline model, full bundling is the only equilibrium irrespective of $W$ when $N \geq N^{*}$. The following proposition provides a complete characterization of when full bundling emerges as an equilibrium.

Proposition 4. Assume that (7) holds. There exists $\bar{N}(W)$ (increasing in $J$ and going to $\infty$ as $J$ grows large) such that full bundling is the only equilibrium when $N \leq$ $\hat{N}(W)$ or $N \geq \bar{N}(W)$, and whenever $\hat{N}(W)<\bar{N}(W)$ it is not an equilibrium for any $N \in(\hat{N}(W), \bar{N}(W))$. Moreover, for any $N \geq N^{*}$, the full bundling is the only equilibrium.

We can now characterize more generally the scope of the bundler's dilemma in this setting. As long as the payoff from offering the full bundle of size $J$ is lower than $(J-1) x$, banks would be collectively better off by removing one lemon from the bundle, thereby leading to a bundler's dilemma.

Corollary 2. Suppose that (7) holds and $N \geq \bar{N}(W)$. There is a bundler's dilemma if and only if $\pi(J)<(J-1) x$. 


\subsection{Fixed number of signals}

In our baseline model, investors sample each bundle once so that as one increases the number of banks, investors are bound to sample more bundles. Alternatively, one may assume that the sampling capacity of investors is fixed independently of the number of bundles, say each investor can sample at most $B$ bundles. Suppose that, conditional on sampling, investors sample one asset uniformly over all assets in the bundle (as in the baseline model), and that they do not trade a bundle they have not sampled irrespective of its price. In this alternative formulation, we can show that Proposition 2 holds provided that both $N$ and $B$ are sufficiently large. The key ingredient for our results is that investors are able to compare across sufficiently many bundles.

Proposition 5. Suppose that each investor can sample at most B bundles and never trades a bundle that is not sampled. There exist $B^{*}$ and $N^{*}$ set independently of $W$ such that if $N \geq N^{*}$ and $B \geq B^{*}$, then full bundling is the only equilibrium.

Showing the equivalent of the bundler's dilemma result in Corollary 1 in this modified setting is immediate, noticing that when $B<N$, each bundle would get at most $\left(1-\left(\frac{1}{J}\right)^{B}\right) / N$. Specifically, we have a bundler's dilemma when $N \geq N^{*}, B \geq B^{*}$, and

$$
\frac{W}{N} \in\left(J x_{1}, \frac{\sum_{j} x_{j}}{1-\left(\frac{1}{J}\right)^{B}}\right) .
$$

Similarly, in the cherry/lemons context described by (7), our analysis can be extended in a straightforward way by noting that when $B<N$, the payoff in (8) is written as

$$
\pi(J)=\min \left(J x,\left(1-\left(\frac{J-1}{J}\right)^{B}\right) \frac{W}{N}\right)
$$

\section{ShORT-SELLiNg AND RATIONAL INVESTORS}

We now investigate the robustness of our main findings to the introduction of shortselling and of rational investors. Questions of interest are the following. (i) Can the bundler's dilemma arise in the presence of permissive short-selling constraints for sampling investors? (ii) Can the bundler's dilemma arise in the presence of very wealthy rational investors?

\subsection{Short-selling}

We assume that investors can use their wealth both for buying and for short-selling. In particular, an investor's trading capacity can be defined as $(w, s)$, meaning that such an investor can use his budget to buy $w / p$ units of an asset of price $p$ or short-sell $s / p$ units of the same asset. The severity of short-selling constraints can be measured by $s$. While the baseline model with no short-selling corresponds to $s=0$, we now consider the case 
in which $s=w$, which corresponds to the case in which the constraints on buying and short-selling are symmetric. Accordingly, the aggregate short-selling capacity is

$$
S=W
$$

In order to determine market clearing prices with short-selling, the supply of a bundle $\alpha_{r}^{i}$ defined in (2) should be modified as

$$
S\left(\alpha_{r}^{i}\right)=\left|\alpha_{r}^{i}\right|+\frac{S}{p\left(\alpha_{r}^{i}\right)} \sum_{k} \eta_{k} \lambda_{k}\left(\alpha_{r}^{i}\right),
$$

where $\lambda_{k}\left(\alpha_{r}^{i}\right) \in[0,1]$ is the fraction of the short-selling capacity of investors with valuations $\widetilde{x}_{k}$ used to short-sell bundle $\alpha_{r}^{i}$.

In order to show that a bundler's dilemma can arise even with permissive shortselling constraints as defined in (11), we specialize the set of assets $X$ as in (7). That is, every bank is endowed with one cherry worth $x$ and $J-1$ lemons worth 0 .

We first observe that condition (11) implies that, irrespective of $W$, the unitary price of each bundle $p$ cannot strictly exceed $x / 2$. If $p>x / 2$, investors prefer to short-sell a bundle based on a valuation 0 rather than buying another bundle based on a valuation $x$. Hence, the demand for a bundle is determined by those who sample only good assets. For any $N$ and $J$, this fraction cannot exceed the fraction of those who sample at least one bad asset and are then willing to sell, showing that there is excess supply at any $p>x / 2$. This observation is summarized in the following claim.

Claim. Assume that (11) holds. We have $p \leq x / 2$ and so $\pi(J) \leq J x / 2$ irrespective of $W$ and of the bundling strategy.

To set our benchmark, consider first a monopolistic setting, i.e., $N=1$. Suppose the monopolist offers a bundle consisting of the $J-1$ lemons and the good asset. Market clearing at any price $p \in(0, x)$ requires

$$
\frac{1}{J} \frac{W}{p}=\frac{J-1}{J} \frac{S}{p}+J
$$

where the left-hand side is the demand associated to those who sample the good asset and the right-hand side is the supply $(J)$ of the bank augmented by the short sales $\left(\frac{J-1}{J} \frac{S}{p}\right)$ of those who sample a lemon. When $S \geq W$ and for any $J \geq 2$, supply exceeds demand at any positive price. In this case, creating disagreement is detrimental to the bank, and the bank prefers to sell the assets separately.

Proposition 6. Assume that (7) and (11) hold, and suppose $N=1$. Full separation strictly dominates any bundling.

Consider now a setting with $N>1$. When all banks offer a bundle of size $J$, market clearing at any price $p \in(0, x / 2)$ requires

$$
\left(1-\left(\frac{J-1}{J}\right)^{N}\right) \frac{W}{N} \frac{1}{p}=J+\left(\frac{J-1}{J}\right)^{N} \frac{S}{N} \frac{1}{p} .
$$


When $W=S$, the corresponding payoff for each bank is given by

$$
\pi^{S}(J)=\min \left(J x / 2,\left(1-2\left(\frac{J-1}{J}\right)^{N}\right) \frac{W}{N}\right) .
$$

Similarly to the case with no short-selling, define

$$
\hat{N}_{S}(W)=\max \left\{N: J x / 2 \leq\left(1-2\left(\frac{J-1}{J}\right)^{N}\right) \frac{W}{N}\right\}
$$

and observe that when $N \leq \hat{N}_{S}(W)$, full bundling is an equilibrium, as no deviation would allow a bank to obtain more than $J x / 2$, which is the payoff obtained with full bundling. We can also define $\bar{N}_{S}(W)$ such that if $N \geq \bar{N}_{S}(W)$, full bundling is the only equilibrium. As with no short-selling, $\bar{N}_{S}(W)$ decreases in $W$ and $\bar{N}_{S}(W) \rightarrow N_{S}^{*}$ when $W \rightarrow 0$, where

$$
N_{S}^{*}=\min \left\{N:\left(\frac{J-1}{J}\right)^{N-1} \leq \frac{J(N-1)}{2(1-J+J N)}\right\} .
$$

We have the following proposition.

Proposition 7. Assume that (7) and (11) hold. There exists $\bar{N}_{S}(W)$ such that full bundling is the only equilibrium when $N \leq \hat{N}_{S}(W)$ or $N \geq \bar{N}_{S}(W)$, and whenever $\hat{N}_{S}(W)<\bar{N}_{S}(W)$, it is not an equilibrium for any $N \in\left(\hat{N}_{S}(W), \bar{N}_{S}(W)\right)$. For any $N \geq N_{S}^{*}$, full bundling is the only equilibrium.

The result in Proposition 7 should be contrasted with the monopolistic case, in which there is no incentive to bundle when $W=S$. The difference between the monopoly and the oligopoly case is that in the latter case, when an investor samples a good asset from at least one bundle, he is not willing to short-sell any other bundle (even if the sample there is bad), as short-selling is perceived to be less profitable than buying shares of the high valuation bundle. This reinforces our insight obtained in the baseline model that incentives to bundle are increased when the number of banks is large.

It should also be noted that $N_{S}^{*}<N^{*}$, where $N^{*}$ is defined in condition (10) to be the minimal $N$ that makes it unprofitable to deviate and attract all those investors who sample a lemon from all other banks when there is no short-selling. These investors have no other option than staying out of the market when short-selling is forbidden. If short-selling is allowed, instead, attracting those investors is harder, as they must find it profitable to buy the asset of the deviating bank as opposed to short-selling any of the other bundles. Hence, while short-selling decreases the payoff from bundling (investors with low evaluations can drive the price down), it also decreases the payoff from deviations. This is suggestive that full bundling may emerge with short-selling when it cannot without short-selling (think of $N_{S}^{*}<N<N^{*}$ and $W$ small enough).

As for the possibility of a bundler's dilemma with short-selling, remember that the payoff from full bundling $\pi^{S}(J)$ can never exceed $J x / 2$ when $W=S$. Thus, whenever $\pi^{S}(J)<(J-1) x / 2$, following a logic similar to that with no short-selling, it can be shown 
that banks would be collectively better off by removing one lemon and offering each a bundle of size $(J-1)$. This shows that a bundler's dilemma can arise for a large set of prices even when $W=S$. This is formally stated as follows.

Corollary 3. Assume that (7) and (11) hold, and suppose $N \geq \bar{N}_{S}(W)$. There is a bundler's dilemma if and only if $\pi^{S}(J)<(J-1) x / 2$.

The distribution of assets considered in this section allows us to illustrate in the simplest form that a bundler's dilemma can arise even with permissive short-selling constraints. While we expect that similar insights can be obtained outside this simple and clearly special specification, further work is needed to gain a more complete view on the impact of short-selling constraints with a general distribution of assets.

\subsection{Rational investors}

We now introduce some possibly very wealthy rational investors in our model. These investors can perfectly assess the fundamental value of each bundle irrespective of the bundling strategies chosen by the banks, which can be interpreted within our sampling framework as allowing them to make infinitely many draws from each bundle. We denote their aggregate wealth as $W_{R}$ and their aggregate short-selling capacity as $S_{R}$.

We start with the immediate observation that in our setting, rational investors have a stabilizing effect on prices, so if their trading capacity is unlimited (i.e., $W_{R}$ and $S_{R} \rightarrow \infty$ ), prices are always equal to fundamentals. For any given $N$, and irrespective of banks' strategies, rational investors know the fundamental value of each bundle. If their trading capacity is sufficiently large, they would arbitrage away any mispricing. Any price strictly below (above) fundamentals would result in excess demand (supply); hence, market clearing can only occur at prices equal to fundamentals, and banks have no incentive to bundles assets. This is formalized as follows.

Claim. Let $(W, S)$ be the trading capacity of the sampling investors. For every $N$, there exists $\left(\bar{W}_{R}, \bar{S}_{R}\right)$ such that for all $W_{R}>\bar{W}_{R}$ and $S_{R}>\bar{S}_{R}$, prices are equal to fundamentals, irrespective of banks' strategies.

We next explore whether when rational investors face tough short-selling constraints, a bundler's dilemma can arise despite the presence of rational investors. So as to illustrate this possibility, consider again the set of assets $X$ as in (7). We note that whenever prices are above fundamentals in the context of Corollary 2, having wealthy rational investors (with no short-selling capacity) would not affect the equilibrium analysis, since such investors would assess that assets are overvalued and would thus prefer to stay out of the market. This simple observation implies the following corollary.

Corollary 4. Assume that (7) holds, $S_{R}=S=0$, and $N \geq \bar{N}(W)$. Irrespective of $W_{R}>0$, there is a bundler's dilemma whenever $\pi(J) \in(x,(J-1) x)$. 


\section{LOAN ORIGINATION}

In this section, we modify our baseline model of Section 2 and introduce the possibility for banks to originate new loans. Specifically, we specialize the initial set of assets as

$$
X_{0}=\left\{x_{j}=0 \text { for } j \leq J_{0}-1 \text {, and } x_{J_{0}}=x>0\right\},
$$

which is the same as in (7), with $J_{0}$ denoting the initial number of loans. We then assume that banks can originate lemons at unitary $\operatorname{cost} c>0$, which can be interpreted as the cost of processing a new loan. In this setting, the only reason for banks to originate lemons is to pool them with the good asset.

\subsection{Monopoly}

To set our benchmark, consider first a monopolistic case with $N=1$. The payoff from offering a bundle with $J$ assets is given by

$$
\pi_{M}(J)=\min (J x, W / J)-c\left(J-J_{0}\right)^{+},
$$

where $\left(J-J_{0}\right)^{+}=\max \left(J-J_{0}, 0\right)$ and $J-J_{0}$ corresponds to the new loans that are originated and pooled. Similarly to the baseline setting, bundling dominates separation if and only if $\pi_{M}(2)>x$. The bank offers a bundle of size $J_{M}$, where $J_{M}=$ $\arg \max _{J \in \mathbb{N}} \pi_{M}(J)$.

\subsection{Oligopoly}

Consider now a setting with $N>1$. Since banks originate loans only to pool them, they would not originate any extra loan if offering a pool of size $J_{0}$ (i.e., pooling all the assets they are endowed with) were not an equilibrium when loan originations are not allowed, i.e., $c=\infty$. Hence, from now on, we assume that

$$
N \leq \hat{N}(W) \quad \text { or } \quad N \geq \bar{N}(W) \quad \text { at } J=J_{0},
$$

where $\hat{N}(W)$ and $\bar{N}(W)$ are defined in Proposition 4.

Denote with $\pi_{D}\left(J_{D}, J\right)$ the payoff of a bank offering a pool of size $J_{D}$ when all other banks offer a pool of size $J$. In a symmetric pure strategy equilibrium (we will show it exists), every bank offers $\tilde{J}$ loans, where

$$
\tilde{J}=\arg \max _{J_{D} \in \mathbb{N}} \pi_{D}\left(J_{D}, \tilde{J}\right) .
$$

Our interest is in finding out $\tilde{J}$ and in how it compares to an efficient determination of the number of loans. Our first result, shown in the Appendix, is that if originating one extra loan is not profitable, then it cannot be profitable to originate any larger number of loans. It is then useful to define the payoff from originating one extra loan when all other banks offer a bundle of size $J$, that is,

$$
\Pi(J)=\pi_{D}(J+1, J)-\pi_{D}(J)
$$


where $\pi_{D}(J)$ is the payoff of every bank when all banks offer a bundle of size $J$ (it is a notational simplification of $\left.\pi_{D}(J, J)\right)$.

One can show that $\Pi(J) \leq x-c$ for all $J$, so it is never profitable to originate a new loan if $c>x$. Hence, from now on, we assume that

$$
c<x \text {. }
$$

Moreover, one can show that $\Pi(J)$ is decreasing in $J$ when $\Pi(J) \geq 0$. Accordingly, let us define

$$
\hat{J}=\min _{J \in \mathbb{N}}\{J: \Pi(J) \leq 0\} .
$$

If banks offer a bundle of size $J<\hat{J}$, then by definition of $\hat{J}$ deviating and offering a bundle of size $J+1$ is profitable, implying that there is no equilibrium in which all banks offer a bundle of size $J<\hat{J}$. If banks offer a bundle of size $J=\hat{J}$, then we can show that $\pi_{D}\left(J_{D}, \hat{J}\right)<\pi_{D}(J)$ for all $J_{D} \neq \hat{J}$, implying that banks offering a bundle of size $\hat{J}$ define an equilibrium. This is stated in the next proposition. ${ }^{16}$

Proposition 8. All banks offering a bundle of size $\hat{J}$ as defined in (14) constitute a symmetric equilibrium. There is no symmetric equilibrium in which all banks offer a bundle of size $J<\hat{J}$.

\subsection{Excessive origination}

Our main interest lies in comparing the equilibrium number of loans $\hat{J}$ with the number of loans that maximize the joint payoff for the banks, denoted as $J^{*}$ and defined by

$$
J^{*}=\arg \max _{J \in \mathbb{N}} \pi(J)-c\left(J-J_{0}\right)^{+},
$$

where $\pi(J)$ is defined in expression (8). We first observe that when other banks offer bundles of size $J<J^{*}$, the marginal benefit of originating and bundling an extra asset is $x$, which exceeds the origination cost. Hence, there is no $J<J^{*}$ that corresponds to a symmetric equilibrium, implying that $\hat{J} \geq J^{*}$. More precisely, we have $\hat{J}=J^{*}$ if and only if $\Pi\left(J^{*}\right) \leq 0$ and $J^{*}>J_{0}$. If instead $\Pi\left(J^{*}\right)>0$ or if $J^{*}<J_{0}$, then $\hat{J}>J^{*}$ and $\hat{J}$ is defined by (14). The following proposition is a summary.

Proposition 9. We have $\hat{J} \geq J^{*}$ for all $c$ and $J_{0}$. We have $\hat{J}>J^{*}$ when $J_{0}>J^{*}$ or when $\Pi\left(J^{*}\right)>0$.

The proposition shows that the possibility for banks to originate and pool low quality loans leads to a collective inefficiency from the banks' perspective. The number of lemons that are sold in equilibrium exceeds not only those offered by a monopolistic

\footnotetext{
${ }^{16}$ The proposition rules out equilibria with $J<\hat{J}$ but not with $J>\hat{J}$. This is sufficient for our purpose to show that even at $\hat{J}$, there is excessive loan origination (see Section 6.3).
} 
bank, but also what oligopolistic banks would choose if they could make a joint decision. ${ }^{17}$ If banks could collectively commit, they would rather originate fewer loans, enjoy higher payoffs, and save the origination costs.

While similar in flavor, this form of collective inefficiency is distinct from the bundler's dilemma highlighted in our previous analysis. First, the inefficiency related to excessive loan origination occurs even when investors are very wealthy. Second, it has different welfare implications. The bundler's dilemma in our baseline model was only affecting asset prices. Given that we assume quasi-linear preferences and that, within the model, prices have no other consequence than a wealth transfer between banks and investors, distorted prices do not affect aggregate welfare, i.e., the sum of investors' welfare and banks' profits. Loan origination, instead, induces pure welfare losses due to the origination costs. We now show that even if those costs are small, aggregate welfare losses can be substantial if the number of banks is large enough.

Let us define total welfare losses due to loan origination as

$$
L=N c\left(\hat{J}-J_{0}\right) .
$$

Consider the case of vanishing origination costs (i.e., $c \rightarrow 0$ ). It is immediate to see that when the cost is arbitrarily small, banks are induced to originate new loans up to the point where full bundling is an equilibrium. As already mentioned, there is no incentive to originate a loan if not for pooling purposes. From Proposition 4, full bundling is an equilibrium when $N \geq \bar{N}(W)$, where $\bar{N}(W)$ increases in $J$. We can define

$$
\bar{J}=\max _{J \in \mathbb{N}}\{J: N \geq \bar{N}(W)\}
$$

and observe that $\hat{J}$ tends to $\bar{J}$ as $c$ tends to 0 . By definition, $\bar{J}$ is the maximal number of loans that are offered in equilibrium even if the cost of generating new loans were arbitrarily small. Even if endowed with $\bar{J}+1$ assets, banks would not all offer a bundle of size $\bar{J}+1$ in equilibrium, since $N<\bar{N}(W)$ at $J=\bar{J}+1$. Such a maximal number of loans is finite when $N$ is finite. As observed in Proposition $4, \bar{N}(W) \rightarrow \infty$ as $J \rightarrow \infty$, implying that $\bar{J}$ remains bounded for any given $N$. It follows that fixing $N$ and letting $c \rightarrow 0$, we can write the welfare loss as $L=N c\left(\bar{J}-J_{0}\right)$ and notice that $L \rightarrow 0$ as $c \rightarrow 0$ since $\bar{J}<\infty$. At the same time, we have that $\bar{J} \rightarrow \infty$ as $N \rightarrow \infty$. Hence, if one lets $c=\kappa / N$ for some constant $\kappa>0$ and lets $N \rightarrow \infty$, then we have $L=\kappa\left(\bar{J}-J_{0}\right)$ and $L \rightarrow \infty$ since $\bar{J} \rightarrow \infty$. This suggests that even if origination costs get small, welfare losses can be significant in a market with a large number of banks.

\section{CONCLUSION}

We have studied banks' incentives to package assets into composite pools when investors base their assessments on a limited sample of the assets in the pool. While we have focused on a specific heuristic of investors and a specific financial instrument for banks, we believe our approach can be viewed as representative of a more general theme

\footnotetext{
${ }^{17}$ Notice that $N J^{*}>J_{M}$ since $J x<\left(1-\left(\frac{J-1}{J}\right)^{N}\right) \frac{W}{N}$ at $J=J_{M}$ and so $N \hat{J}>J_{M}$.
} 
in which investors use simple valuation models-for example, models that worked well for similar yet more familiar products-and product complexity is endogenous.

Our analysis could be extended to explore the incentives for financial institutions to create complexity when investors use other heuristics as well as to investigate other forms of complexity. Investors may find it hard to evaluate financial products not only because of the heterogeneity of the underlying assets as in our model, but also because of the complex mapping between the value of the underlying assets and the payoff to investors (as, for example, in mortgage-backed securities with complex tranching structure or in several other structured products).

Complexity would also be amplified if, on top of average expected values, investors were to assess other characteristics of the assets. Under risk aversion, for example, investors would care about correlations across assets, which can be difficult to evaluate. Misunderstanding of correlations could be another source of investment errors (such considerations have been a central theme in the recent financial crisis (Coval et al. (2009))). We believe that extending our model to allow banks to offer more general securities and investors to care about the variance in asset payoffs is an interesting avenue for future research.

\section{APpendix: Proofs}

Proof of Proposition 1. Supposing $W>\max \left(2\left(x_{2}+x_{1}\right), \sum_{j} x_{j}\right)$, full separation gives $\sum_{j} x_{j}$. Suppose the bank bundles assets $\left\{X_{1}, X_{2}\right\}$ and sells the other assets separately. Consider first a candidate equilibrium in which investors who sample $x_{2}$ from the bundle are indifferent between trading the single asset $x_{j}$ and the bundle. That requires $2 x_{2} / p_{2}=x_{j} / p_{j}$ for all $j>2$, where $p_{2}$ is the price of the bundle and $p_{j}$ is the price of the asset $x_{j}$. In addition, we need that $p_{2}+\sum_{j>2} p_{j} \leq W$, so aggregate wealth is enough to buy at prices $p_{2}$ and $p_{j}$. The above conditions give $p_{2} \leq 2 x_{2} W /\left(\sum_{j>2} x_{j}+2 x_{2}\right)$ and $p_{j} \leq x_{j} W /\left(\sum_{j>2} x_{j}+2 x_{2}\right)$. In addition, we need that $p_{2} \leq W / 2$ so that those investors who have valuation $x_{2}$ for the $\left(x_{2}, x_{1}\right)$ bundle can indeed drive the price to $p_{2}$. Supposing $2 x_{2}<\sum_{j>2} x_{j}$, we have $\frac{2 x_{2}}{\sum_{j>2} x_{j}+2 x_{2}}<\frac{W}{2}$, and so $p_{2}=\min \left(2 x_{2}, \frac{2 x_{2}}{\sum_{j>2} x_{j}+2 x_{2}} W\right)$ and $p_{j}=\min \left(x_{j}, \frac{x_{j}}{\sum_{j>2} x_{j}+2 x_{2}} W\right)$ for $j>2$. So the payoff of the bank is

$$
\min \left(W, 2 x_{2}+\sum_{j>2} x_{j}\right),
$$

which exceeds $\sum_{j} x_{j}$. Suppose $2 x_{2} \geq \sum_{j>2} x_{j}$, which can only occur if $J=3$ and $2 x_{2} \geq x_{3}$. Then we must have $p_{2}=W / 2$ and $p_{3}=x_{3} W / 4 x_{2}$. That cannot be in equilibrium since investors who sample $x_{l}$ still have money and would like to drive the price $p_{3}$ up. So if $2 x_{2}>x_{3}$, investors are indifferent only if $p_{2}=2 x_{2}$ and $p_{3}=x_{3}$. That requires $W>4 x_{2}$. If $W<4 x_{2}$, then we must have $p_{2}<2 x_{2} \frac{p_{3}}{x_{3}}$. If $W \in\left(2 x_{3}, 4 x_{2}\right)$, we have $p_{2}=\frac{W}{2}$ and $p_{3}=x_{3}$. If $W<2 x_{3}$, we have $p_{2}=p_{3}=\frac{W}{2}$. The payoff of the bank is

$$
\min \left(W / 2,2 x_{2}\right)+\min \left(W / 2, x_{3}\right),
$$


which also exceeds $\sum_{j} x_{j}$. Suppose $W \leq \max \left(2\left(x_{2}+x_{1}\right), \sum_{j} x_{j}\right)$. If $W \leq \sum_{j} x_{j}$, then no bundling strictly dominates full separation. If $W \in\left(\sum_{j} x_{j}, 2\left(x_{2}+x_{1}\right)\right]$, we must have $\sum_{j} x_{j}<2\left(x_{2}+x_{1}\right)$, which cannot be for $J>3$. For $J=3$ and $W \leq 2\left(x_{2}+x_{1}\right)$, no bundling strictly dominates full separation.

Proof of Lemma 1. Denote with $\mathcal{H}$ the set of (possibly identical) bundles $r \in \arg \min _{r} \mu_{r}$ and denote with $\mathcal{L}$ the set of (possibly identical) bundles $r \notin \arg \min _{r} \mu_{r}$, with $|\mathcal{H}|=H$ and $|\mathcal{L}|=L$. Suppose, by contradiction, (4) is violated. Then $H \geq 1$ and $L \geq 1$, and

$$
\mu_{r}<\mu_{\tilde{r}} \quad \text { for all } r \in \mathcal{H} \text { and all } \tilde{r} \in \mathcal{L} .
$$

Given (15), the $H$ bundles would attract at least all those investors who sample $x_{r}^{*}$ from at least one bundle $r \in \mathcal{H}$, and so at least

$$
W_{\hat{r}}=\left(1-\prod_{r \in \mathcal{H}}\left(\frac{J_{r}-1}{J_{r}}\right)\right) W .
$$

The other bundles would attract at most the remaining wealth $W-W_{\hat{r}}$. Denoting with $\hat{r} \in \mathcal{H}$ the bundle that receives the largest fraction of $W_{\hat{r}}$, it would attract at least $1 / \mathrm{H}$ of it. Similarly, denoting with $\bar{r} \in \mathcal{L}$ the bundle that receives the lowest fraction of $W-W_{\hat{r}}$, it would attract at most $1 / L$ of it. This implies that

$$
p_{\hat{r}} \geq \min \left(x_{\hat{r}}^{*}, \frac{1}{H} \frac{W_{\hat{r}}}{J_{\hat{r}}}\right)
$$

and

$$
p_{\bar{r}} \leq \frac{1}{L} \frac{W-W_{\hat{r}}}{J_{\bar{r}}}
$$

Notice that if

$$
x_{\hat{r}}^{*} \leq \frac{1}{H} \frac{W_{\hat{r}}}{J_{\hat{r}}},
$$

then $\mu_{\hat{r}}=1$ and so $\mu_{\hat{r}}<\mu_{\bar{r}}$ would imply $p_{\bar{r}}>x_{\bar{r}}^{*}$, which violates market clearing. Hence, $\mu_{\hat{r}}<\mu_{\bar{r}}$ requires

$$
\frac{1}{H} \frac{W_{\hat{r}}}{J_{\hat{r}}} \frac{1}{x_{\hat{r}}^{*}}<\frac{1}{L} \frac{W-W_{\hat{r}}}{J_{\bar{r}}} \frac{1}{x_{\bar{r}}^{*}},
$$

which gives

$$
L<\frac{J_{\hat{r}}}{J_{\bar{r}}} \frac{x_{\hat{r}}^{*}}{x_{\bar{r}}^{*}} \frac{W-W_{\hat{r}}}{W_{\hat{r}}} H .
$$

Notice that the right-hand side of (16) decreases in $H$ and tends to 0 as $H \rightarrow \infty$. In fact, we can write

$$
\prod_{r \in \mathcal{H}}\left(\frac{J_{r}-1}{J_{r}}\right)=z^{H}
$$


for some $z \in(0,1)$ and so

$$
\frac{W-W_{\hat{r}}}{W_{\hat{r}}} H=\frac{z^{H}}{1-z^{H}} H .
$$

We notice that the right-hand side of (17) decreases in $H$ if and only if $H \ln z-z^{H}+1<0$, that $H \ln z-z^{H}+1$ decreases in $H$, and that $\ln z-z+1<0$ for all $z \in(0,1)$. Hence, condition (16) is violated if either $H$ or $L$ (or both) grow sufficiently large, which must be the case when $N \rightarrow \infty$ since $H+L \geq N$ (the total number of bundles cannot fall short of the number of banks). Hence, there exists an $N_{0}$ such that (4) must hold for $N \geq N_{0}$, which proves our result.

Proof of Proposition 2. We first show that full bundling is an equilibrium when $N$ is large. We then show that no other bundling can be an equilibrium when $N$ is large.

Part 1: If $N$ is sufficiently large, full bundling in an equilibrium for all $W$.

Suppose all banks offer the full bundling and denote with $\pi^{F}$ the payoff for each bank. If $\left(1-\left(\frac{J-1}{J}\right)^{N}\right) \frac{W}{N} \geq J x_{J}$, then $\pi^{F}=J x_{J}$ and no other bundling can increase banks' payoffs. Suppose then

$$
\left(1-\left(\frac{J-1}{J}\right)^{N}\right) \frac{W}{N}<J x_{J}
$$

We have

$$
\pi^{F} \geq\left(1-\left(\frac{J-1}{J}\right)^{N}\right) \frac{W}{N}
$$

The condition is derived by noticing that each bundle attracts at least those who sample the maximal asset in the bundle $x_{J}$. These investors are willing to invest all their wealth, since the price of the bundle is strictly lower than their evaluation. From (18), the price of the bundle is lower than $x_{J}$.

Suppose bank $j$ deviates and offers at least two bundles, indexed by $r$. The payoff of the deviating bank is $\pi^{j}=\sum_{r \geq 1} J_{r} p_{r}$. From (4) we have

$$
\pi^{j}=\mu_{0} \sum_{r \geq 1} J_{r} x_{r}^{*}
$$

and so

$$
\pi^{j} \leq \mu_{0}(J-1) x_{J}+\mu_{0} x_{J-1} .
$$

Notice also that $\pi^{j}+(N-1) J \mu_{0} x_{J} \leq W$ and since from (20), $\pi^{j} \geq \mu_{0} \sum_{j} x_{j}$, we have

$$
\mu_{0} \leq \frac{W}{\sum_{j} x_{j}+(N-1) J x_{J}} .
$$

Together with (21), that gives

$$
\pi^{j} \leq \frac{\left((J-1) x_{J}+x_{J-1}\right) W}{\sum_{j} x_{j}+(N-1) J x_{J}} .
$$


So as to show that the deviation is not profitable, given (19), it is enough to show that

$$
\frac{\left((J-1) x_{J}+x_{J-1}\right) W}{\sum_{j} x_{j}+(N-1) J x_{J}}<\left(1-\left(\frac{J-1}{J}\right)^{N}\right) \frac{W}{N} .
$$

Equation (22) can be written as

$$
\left(\frac{J-1}{J}\right)^{N}<\frac{\sum_{j} x_{j}-J x_{J}+N\left(x_{J}-x_{J-1}\right)}{\sum_{j} x_{j}+(N-1) J x_{J}} .
$$

Notice that the left-hand side of (23) decreases monotonically in $N$ and tends to 0 as $N \rightarrow \infty$, while the right-hand side of (23) increases monotonically in $N$ and tends to

$$
\frac{x_{J}-x_{J-1}}{J x_{J}}>0
$$

as $N \rightarrow \infty$. Hence, $\pi^{j}<\pi^{F}$ and so full bundling is an equilibrium for $N$ sufficiently large.

Part 2: If $N$ is sufficiently large and irrespective of $W$, no alternative bundling is an equilibrium.

Suppose there is one bank, say bank $j$, that offers at least two bundles and it deviates by offering the full bundle. From (4), the payoff of the deviating bank can be written as $\mu_{0} J x_{J}$. If $\mu_{0}=1$, then the deviation is profitable, since any other bundling would give strictly less than $J x_{J}$. Suppose then $\mu_{0}<1$. As the price of each bundle is strictly lower than the best asset from the bundle, those who sample $x_{r}^{*}$ from at least one bundle $r$ will invest all their wealth. The total amount of wealth invested is then at least

$$
\hat{W}=\left(1-\prod_{r}\left(\frac{J_{r}-1}{J_{r}}\right)^{M}\right) W,
$$

where $M$ is the number of bundles offered after the deviation. Since $M \geq N$ and $J_{r} \leq J$ for all $r$, we have

$$
\hat{W} \geq\left(1-\left(\frac{J-1}{J}\right)^{N}\right) W .
$$

Consider first a candidate symmetric equilibria in which the payoff of the nondeviating banks is the same and it is denoted by $\pi^{-j}$. By definition we have $\pi^{j}+(N-1) \pi^{-j} \geq$ $\hat{W}$ and $\pi^{-j} \leq \mu_{0} x_{J-1}+(J-1) \mu_{0} x_{J}$, which gives $\mu_{0} J x_{J}+(N-1) \mu_{0} x_{J-1}+(N-1)(J-$ 1) $\mu_{0} x_{J} \geq \hat{W}$. Hence,

$$
\mu_{0} \geq \frac{\hat{W}}{J x_{J}+(N-1) x_{J-1}+(N-1)(J-1) x_{J}}
$$

and so

$$
\pi^{j} \geq \frac{\hat{W} J x_{J}}{J x_{J}+(N-1) x_{J-1}+(N-1)(J-1) x_{J}} .
$$


Since the payoff before deviation was at most $W / N$, the deviation is profitable if

$$
\frac{J x_{J}\left(1-\left(\frac{J-1}{J}\right)^{N}\right) W}{J x_{J}+(N-1) x_{J-1}+(N-1)(J-1) x_{J}}>\frac{W}{N},
$$

which is written as

$$
x_{J}-x_{J-1}>\frac{N}{N-1}\left(\frac{J-1}{J}\right)^{N} J x_{J}
$$

and that shows that $\pi^{j}>\pi^{F}$ and so the alternative bundling is not an equilibrium for $N$ sufficiently large.

Suppose there exists an asymmetric equilibrium. Denoting with $\pi^{j}$ the payoff of a generic bank $j$ in such an equilibrium, we must have $\min _{j} \pi^{j}<\max _{j} \pi^{j}$. Consider bank $\tilde{j} \in \arg \min _{j} \pi^{j}$. Suppose bank $\tilde{j}$ deviates and offers the full bundle. From the above argument, its payoff after deviation would be at least $\hat{W} / N$, and from (24), $\hat{W} \rightarrow W$ as $N \rightarrow \infty$. Since $\tilde{j} \in \arg \min _{j} \pi^{j}$, we have $\pi^{\tilde{j}}<W / N$. Hence, $\pi^{\tilde{j}}<\hat{W} / N$ for $N$ sufficiently large, which rules out the possibility of asymmetric equilibria when $N$ is large.

Proof of Proposition 3. Denote with $r$ the bundles offered by a generic bank $j$. Its payoff can be written as $\pi^{j}=\sum_{r \geq 1} J_{r} p_{r}$, and from (4), we have

$$
\pi^{j}=\mu_{0} \sum_{r \geq 1} J_{r} x_{r}^{*}
$$

for some $\mu_{0}$. If all other banks offer a partition $\alpha=\left\{\alpha_{f}\right\}_{f}$, we have

$$
\mu_{0}^{F}\left(\sum_{r \geq 1} J_{r} x_{r}^{*}+(N-1) \sum_{f \geq 1} J_{f} x_{f}^{*}\right)=W^{F}
$$

for some $\mu_{0}^{F}$ and where $W^{F}$ is the total amount of wealth invested. Supposing instead that the other banks offer a partition $\tilde{\alpha}=\left\{\alpha_{c}\right\}_{c}$, which is coarser than $\alpha$, we have

$$
\mu_{0}^{C}\left(\sum_{r \geq 1} J_{r} x_{r}^{*}+(N-1) \sum_{c \geq 1} J_{c} x_{c}^{*}\right)=W^{C}
$$

for some $\mu_{0}^{C}$ and $W^{C}$. Supposing that $\mu_{0}^{C} \geq \mu_{0}^{F}$, then we must have $W^{C} \leq W^{F}$. The fraction of wealth that is not invested corresponds to the probability that an investor samples an asset with value lower than the price from all bundles. This probability cannot be larger in $\alpha$ than in $\tilde{\alpha}$. By definition, there exists at least one element $\tilde{\alpha}_{c} \in \tilde{\alpha}$ that is obtained by the union of at least two elements $\tilde{\alpha}_{f}, \hat{\alpha}_{f} \in \alpha$. Hence, if $\mu_{0}^{C} \geq \mu_{0}^{F}$, the probability of sampling an asset whose value is lower than the price in $\tilde{\alpha}_{c}$ cannot be lower than the probability of sampling such an asset both in $\tilde{\alpha}_{f}$ and in $\hat{\alpha}_{f}$. Notice that from (26) and (27) that $\mu_{0}^{C} \geq \mu_{0}^{F}$ contradicts $W^{C} \leq W^{F}$, since, by definition, $\sum_{c \geq 1} J_{c} x_{c}^{*}>\sum_{f \geq 1} J_{f} x_{f}^{*}$. Hence, we must have $\mu_{0}^{C}<\mu_{0}^{F}$, and from (25), this shows that bank $j$ receives a higher payoff when the other banks offer a finer partition. 
Proof of Proposition 4. Let us first show when full bundling is an equilibrium. Suppose all banks offer the full bundle and the price is $p=x$. As prices cannot increase further, there is no profitable deviation. We have $p=x$ when

$$
J x \leq\left(1-\left(\frac{J-1}{J}\right)^{N}\right) \frac{W}{N},
$$

which can be written as $N \leq \hat{N}(W)$. Suppose $p \in(0, x)$, and a bank deviates and sells a bundle of size $J_{D}$. Denote with $p_{D}$ the unitary price of the deviating bank and with $\tilde{p}$ the unitary price of the other banks (all nondeviating banks should be traded at the same price or markets would not clear). ${ }^{18}$ Supposing that $p_{D}<\tilde{p}$, market clearing for the deviating bank requires

$$
\frac{1}{J_{D}} \frac{W}{p_{D}}=J_{D}
$$

while market clearing for the other banks requires

$$
\left(1-\frac{1}{J_{D}}-\frac{J_{D}-1}{J_{D}}\left(\frac{J-1}{J}\right)^{N-1}\right) \frac{W}{(N-1) \tilde{p}}=J .
$$

Condition $p_{D}<\tilde{p}$ requires

$$
\min \left(x, \frac{1}{J_{D}} \frac{1}{J_{D}} W\right)<\min \left(x,\left(1-\frac{1}{J_{D}}-\frac{J_{D}-1}{J_{D}}\left(\frac{J-1}{J}\right)^{N-1}\right) \frac{W}{(N-1)} \frac{1}{J}\right) .
$$

Notice that the condition can be satisfied only if $x>\frac{1}{J_{D}} \frac{1}{J_{D}} W$, and so $p_{D}<\tilde{p}$ requires, in particular,

$$
\frac{1}{J_{D}} \frac{1}{J_{D}}<\left(1-\frac{1}{J_{D}}-\frac{J_{D}-1}{J_{D}}\left(\frac{J-1}{J}\right)^{N-1}\right) \frac{1}{(N-1)} \frac{1}{J}
$$

that is,

$$
\left(\frac{J-1}{J}\right)^{N-1}<1-\frac{J(N-1)}{J_{D}^{2}-J_{D}}
$$

The payoff of the deviating bank is $W / J_{D}$, so the deviation is profitable only if

$$
\frac{1}{J_{D}}>\left(1-\left(\frac{J-1}{J}\right)^{N}\right) \frac{1}{N}
$$

that is,

$$
\left(\frac{J-1}{J}\right)^{N-1}>\left(1-\frac{N}{J_{D}}\right) \frac{J}{J-1}
$$

\footnotetext{
${ }^{18}$ Suppose there is a group of $M \geq 1$ nondeviating banks with $p=p_{1}$ and some nondeviating bank with $p=p_{2}$ and $p_{2}>p_{1}$. The demand for a bank with price $p_{1}$ is at least $\frac{1}{J}\left(\frac{J-1}{J}\right)^{M-1} P$, where $P$ is the probability of drawing no good asset from a bundle with price $p<p_{1}$, while the demand for a bank with price $p_{2}$ is at most $\frac{1}{J}\left(\frac{J-1}{J}\right)^{M} P$, which cannot exceed the demand of a bank with price $p_{1}$, thereby contradicting that $p_{2}>p_{1}$.
} 
Conditions (29) and (30) require

$$
\left(J-J_{D}\right)\left(J+J_{D}-J N-1\right)>0 .
$$

Since nondeviating banks are bundling all their assets, we have $J>J_{D}$, and so conditions (29) and (30) require $J+J_{D}-J N-1>0$, which is violated for any $N \geq 2$ and $J>J_{D}$. Hence, we cannot have a profitable deviation with $p_{D}<\tilde{p}$.

Suppose instead that $p_{D}=\tilde{p}$. The payoff of the deviating bank is $\min \left(J_{D} x\right.$, $\left.\hat{\pi}_{D}\left(J_{D}, J\right), W / J_{D}\right)$, with

$$
\hat{\pi}_{D}\left(J_{D}, J\right)=\frac{J_{D}}{J_{D}+(N-1) J}\left(1-\frac{J_{D}-1}{J_{D}}\left(\frac{J-1}{J}\right)^{N-1}\right) W .
$$

Total demand corresponds to those investors who sample the good asset from at least one bundle (this is a fraction $1-\frac{J_{D}-1}{J_{D}}\left(\frac{J-1}{J}\right)^{N-1}$ of the investors), and each bundle receives a fraction of the demand in proportion to its size (so the deviating banks receive a fraction $\left.\frac{J_{D}}{J_{D}+(N-1) J}\right)$. We now show that

$$
\hat{\pi}\left(J_{D}, J\right)>\pi(J) \text { if and only if } J_{D}>J .
$$

This means that deviation to any $J_{D}<J$ is not profitable when $p_{D}=\tilde{p}$. To see this, notice that $\hat{\pi}\left(J_{D}, J\right)>\pi(J)$ can be rewritten as $\left(\frac{J-1}{J}\right)^{N-1}\left(J-J_{D}\right)>\left(J-J_{D}\right) \frac{(N-1) J}{(J N-J+1)}$. If $J>J_{D}$, this is written as $\left(\frac{J-1}{J}\right)^{N-1}>\frac{(N-1) J}{(J N-J+1)}$, which is violated for all $J$ when $N=2$ and this is a fortiori true for any larger $N$. Hence, there is no profitable deviation for the deviating bank with $p_{D}=\tilde{p}$.

Finally, supposing that $p_{D}>\tilde{p}$, market clearing for the deviating bank requires

$$
\frac{1}{J_{D}}\left(\frac{J-1}{J}\right)^{N-1} \frac{W}{p_{D}}=J_{D},
$$

as the demand comes only from those who draw a good asset for the deviating bank and a lemon from all other banks, while market clearing for the other banks requires

$$
\frac{1}{N-1}\left(1-\left(\frac{J-1}{J}\right)^{N-1}\right) \frac{W}{\tilde{p}}=J .
$$

Condition $p_{D}>\tilde{p}$ requires

$$
\min \left(x, \frac{1}{J_{D}} \frac{1}{J_{D}}\left(\frac{J-1}{J}\right)^{N-1} W\right)>\min \left(x, \frac{W}{N-1} \frac{1}{J}\left(1-\left(\frac{J-1}{J}\right)^{N-1}\right)\right) .
$$

The deviation is profitable if and only if

$$
\min \left(J_{D} x, \frac{1}{J_{D}}\left(\frac{J-1}{J}\right)^{N-1} W\right)>\min \left(J x,\left(1-\left(\frac{J-1}{J}\right)^{N}\right) \frac{W}{N}\right) .
$$

Notice that

$$
\frac{J_{D}}{J}\left(1-\left(\frac{J-1}{J}\right)^{N-1}\right) \frac{W}{N-1}<\left(1-\left(\frac{J-1}{J}\right)^{N}\right) \frac{W}{N}
$$


since at $J_{D}=J-1$ that is equivalent to $N-J+J\left(\frac{1}{J}(J-1)\right)^{N}>0$, which is the case for $N=2$, and it is increasing in $N$. Hence, condition (32) implies condition (31). Defining

$$
\hat{J}_{D}=\left(\frac{1}{x}\left(\frac{J-1}{J}\right)^{N-1} W\right)^{1 / 2}
$$

we have $J_{D}^{*}=1$ if $\hat{J}_{D} \leq 1, J_{D}^{*}=\hat{J}_{D}$ if $\hat{J}_{D} \in(1, J-1)$, and $J_{D}^{*}=J-1$ if $\hat{J}_{D} \geq J-1$. Notice that $J_{D}^{*} \leq 1$ is written as

$$
\left(\frac{J-1}{J}\right)^{N-1} \leq \frac{x}{W}
$$

and $J_{D}^{*} \geq J-1$ is written as

$$
\left(\frac{J-1}{J}\right)^{N-1}(J-1)^{-2} \geq \frac{x}{W} .
$$

If (33) holds, $J_{D}^{*}=1$ and the deviation is profitable if and only if

$$
\left(\frac{J-1}{J}\right)^{N-1} W>\left(1-\left(\frac{J-1}{J}\right)^{N}\right) \frac{W}{N}
$$

that is,

$$
\left(\frac{J-1}{J}\right)^{N-1} N+\left(\frac{J-1}{J}\right)^{N}>1
$$

If (34) holds, $J_{D}^{*}=J-1$ and the deviation is profitable if and only if

$$
(J-1) x>\left(1-\left(\frac{J-1}{J}\right)^{N}\right) \frac{W}{N},
$$

that is,

$$
\frac{N}{W}(J-1) x+\left(\frac{J-1}{J}\right)^{N}>1 .
$$

If both (33) and (34) are violated, $J_{D}^{*}=\hat{J}_{D}$ and the deviation is profitable if and only if

$$
x^{1 / 2}\left(\frac{J-1}{J}\right)^{\frac{N-1}{2}} W^{1 / 2}>\left(1-\left(\frac{J-1}{J}\right)^{N}\right) \frac{W}{N},
$$

that is,

$$
N\left(\frac{x}{W}\right)^{1 / 2}\left(\frac{J-1}{J}\right)^{\frac{N-1}{2}}+\left(\frac{J-1}{J}\right)^{N}>1 .
$$

Combining cases (35), (36), and (37), let us define

$$
\bar{N}(W)=\min \{N: \Phi(N) \leq 1\}
$$


and

$$
\Phi(N)=\left\{\begin{array}{l}
\left(\frac{J-1}{J}\right)^{N-1} N+\left(\frac{J-1}{J}\right)^{N} \\
\quad \text { if }\left(\frac{J-1}{J}\right)^{N-1} \leq \frac{x}{W}, \\
N\left(\frac{x}{W}\right)^{1 / 2}\left(\frac{J-1}{J}\right)^{\frac{N-1}{2}}+\left(\frac{J-1}{J}\right)^{N} \\
\quad \text { if } \frac{x}{W} \in\left(\left(\frac{J-1}{J}\right)^{N-1}(J-1)^{-2},\left(\frac{J-1}{J}\right)^{N-1}\right), \\
\left.\frac{N}{W}(J-1) x+\left(\frac{J-1}{J}\right)^{N}\right) \\
\quad \text { if }\left(\frac{J-1}{J}\right)^{N-1}(J-1)^{-2} \geq \frac{x}{W} .
\end{array}\right.
$$

Combining (9) and (38), we have that full bundling is an equilibrium if $N \leq \hat{N}(W)$ or $N \geq \bar{N}(W)$. Provided that $\bar{N}(W)>\hat{N}(W)$, full bundling is not an equilibrium if $N \in$ $(\hat{N}(W), \bar{N}(W))$.

To see if there are other symmetric equilibria, suppose that all banks offer a bundle of size $J_{1}<J_{0}$ smaller than the full bundle, and suppose a bank deviates and offers a bundle of size $J_{D}>J_{1}$. As shown above, $\hat{\pi}\left(J_{D}, J_{1}\right)>\pi\left(J_{1}\right)$ if and only if $J_{D}>J_{1}$, implying that if $J_{1}<J_{0}$ and $\hat{\pi}\left(J_{1}+1, J_{1}\right)<W /\left(J_{1}+1\right)$, the deviation to $J_{D}=J_{1}+1$ is profitable. Suppose instead that $\hat{\pi}\left(J_{1}+1, J_{1}\right)>W /\left(J_{1}+1\right)$, so by deviating to $J_{D}=J_{1}+1$, the banks gets $W /\left(J_{1}+1\right)$. The deviation is profitable if

$$
\frac{W}{J_{1}+1}>\min \left(J_{1} x,\left(1-\left(\frac{J_{1}-1}{J_{1}}\right)^{N}\right) \frac{W}{N}\right),
$$

which is the case for all $J_{1}$ for $N \geq 3$. To sustain an equilibrium in which all banks offer a bundle of size $J_{1}<J_{0}$, it is then necessary that $N=2$ and $W /\left(J_{1}+1\right)<J_{1} x$, that is,

$$
\frac{x}{W}>\frac{1}{\left(J_{1}+1\right) J_{1}} \geq \frac{1}{J_{0}\left(J_{0}-1\right)} .
$$

It can be shown that $\Phi(N)>1$ when $N=2$ and (39) holds, implying that if $N \geq \bar{N}(W)$, then the deviation to $J_{D}=J_{1}+1$ is profitable and there is no symmetric equilibrium in which all banks offer a bundle of size $J_{1}<J_{0}$.

Proof of Corollary 2. Suppose that $\pi\left(J_{0}\right) \geq\left(J_{0}-1\right) x$. The payoff obtained when all banks remove $J$ lemons from the pool cannot exceed $\left(J_{0}-J\right) x$; hence, it cannot exceed $\left(J_{0}-1\right) x$ no matter what $J$ is. Supposing that $\pi\left(J_{0}\right)<\left(J_{0}-1\right) x$, we have

$$
\left.\pi\left(J_{0}\right)=\left(1-\left(\frac{J_{0}-1}{J_{0}}\right)^{N}\right) \frac{W}{N}\right) .
$$

Supposing that banks were to offer a bundle of size $J_{0}-1$ instead, their payoff would be

$$
\pi\left(J_{0}-1\right)=\min \left(\left(J_{0}-1\right) x,\left(1-\left(\frac{J_{0}-2}{J_{0}-1}\right)^{N}\right) \frac{W}{N}\right),
$$


which is shown to exceed $\pi\left(J_{0}\right)$ when $\pi\left(J_{0}\right)<\left(J_{0}-1\right) x$, given that $\left(\frac{J_{0}-2}{J_{0}-1}\right)^{N}<\left(\frac{J_{0}-1}{J_{0}}\right)^{N}$.

Proof of Proposition 5. Let us first show that when $N$ and $B$ are sufficiently large, Lemma 1 holds. Adopting the notation in the proof of Lemma 1, suppose that $H \leq B$. Then the proof is exactly the same. If $H>B$, the minimal fraction of wealth attracted by the $H$ bundles can be written as $1-z^{B}$ for some $z \in(0,1)$, and so equation (16) is written as

$$
L<\frac{J_{\hat{r}}}{J_{\bar{r}}} \frac{x_{\hat{r}}^{*}}{x_{\bar{r}}^{*}} \frac{z^{B}}{1-z^{B}} H,
$$

which is violated when $B$ is sufficiently large, showing that (4) must hold when $B$ is sufficiently large.

We now show that full bundling is an equilibrium when $B$ is sufficiently large. The proof is exactly as in Proposition 2 when $N \leq B$. If $N>B$, the payoff from full bundling in (19) is written as

$$
\pi^{F} \geq\left(1-\left(\frac{J-1}{J}\right)^{B}\right) \frac{W}{N},
$$

and condition (22) for having no profitable deviations can be written as

$$
\left(\frac{J-1}{J}\right)^{B}<\frac{\sum_{j} x_{j}-J x_{J}+N\left(x_{J}-x_{J-1}\right)}{\sum_{j} x_{j}+(N-1) J x_{J}},
$$

that is, the case when $B$ is sufficiently large.

Finally, we show that no alternative bundling is an equilibrium when $B$ is sufficiently large, again using the same notation as in the proof of Proposition 2 . If $M \leq B$, the proof in unchanged. If $M>B$, (24) is written as

$$
\hat{W} \geq\left(1-\left(\frac{J-1}{J}\right)^{B}\right) W
$$

and the condition for having a profitable deviation is written as

$$
x_{J}-x_{J-1}>\frac{N}{N-1}\left(\frac{J-1}{J}\right)^{B} J x_{J},
$$

showing that the alternative bundling is not an equilibrium for $B$ sufficiently large.

Proof of Proposition 7. First, we show when full bundling is an equilibrium. Suppose all banks offer the full bundle and the price is $p=x / 2$. Then prices cannot increase further and so there is no profitable deviation. We have $p=x / 2$ when

$$
\frac{x}{2} J \leq\left(1-2\left(\frac{J-1}{J}\right)^{N}\right) \frac{W}{N} .
$$


It should be mentioned that in (40), we say that in case of indifference between buying and short-selling, there exists a way to split the orders so as to have $p=x / 2$. This is similar to the treatment of $p=x$ in condition (28) in the absence of short-selling. From (40), full bundling is an equilibrium when $N \leq \hat{N}_{S}(W)$. Suppose that $p \in(0, x / 2)$, and a bank deviates and sells a bundle of size $J_{D}$. Denote with $p_{D}$ the unitary price of the deviating bank and denote with $\tilde{p}$ the unitary price of the other banks (assuming for now it is the same for all nondeviating banks). Supposing that $p_{D}>\tilde{p}$, market clearing for the deviating bank requires

$$
\frac{1}{J_{D}}\left(\frac{J-1}{J}\right)^{N-1} \frac{W}{p_{D}}=J_{D}+\frac{J_{D}-1}{J_{D}}\left(\frac{J-1}{J}\right)^{N-1} \frac{W}{p_{D}},
$$

as the demand comes only from those who draw a good asset for the deviating bank and a lemon from all other banks, while all those who draw a lemon strictly prefer to sell the asset of the deviating bank since $p_{D}>\tilde{p}$, but this is impossible to satisfy with $p_{D}>0$. Hence, there is no market clearing at $p_{D}>\tilde{p}$ and so no profitable deviation, irrespective of $N$.

Supposing instead $p_{D}<\tilde{p}$, we show that even in this case there is no profitable deviation, irrespective of $N$. Since nondeviating banks are bundling all their assets, we have

$$
J_{D}<J
$$

When $p_{D}<\tilde{p}$, market clearing for the deviating bank requires

$$
\frac{1}{J_{D}} \frac{W}{p_{D}}=J_{D}
$$

while market clearing for the other banks requires

$$
\left(1-\frac{1}{J_{D}}-\frac{J_{D}-1}{J_{D}}\left(\frac{J-1}{J}\right)^{N-1}\right) \frac{W}{(N-1) \tilde{p}}=J+\frac{J_{D}-1}{J_{D}}\left(\frac{J-1}{J}\right)^{N-1} \frac{W}{(N-1) \tilde{p}}
$$

and so

$$
\tilde{p}=\left(1-\frac{1}{J_{D}}-2 \frac{J_{D}-1}{J_{D}}\left(\frac{J-1}{J}\right)^{N-1}\right) \frac{W}{(N-1)} \frac{1}{J} .
$$

The payoff of the deviating bank is $W / J_{D}$, so the deviation is profitable if

$$
\frac{W}{J_{D}}>\left(1-2\left(\frac{J-1}{J}\right)^{N}\right) \frac{W}{N}
$$

while $p_{D}<\tilde{p}$ can be written as

$$
\frac{W}{J_{D}}<\left(1-\frac{1}{J_{D}}-2 \frac{J_{D}-1}{J_{D}}\left(\frac{J-1}{J}\right)^{N-1}\right) \frac{W}{(N-1)} \frac{J_{D}}{J} .
$$

Conditions (42) and (43) require that

$$
J-N+N J_{D}-J N+\left(\frac{J-1}{J}\right)^{N-1}\left(2-2 J-2 N J_{D}+2 J N\right)>0 .
$$


We now show that, irrespective of $N$, conditions (41) and (44) cannot be satisfied. Notice that $\tilde{p}>0$ requires

$$
\left(\frac{J-1}{J}\right)^{N-1}<1 / 2
$$

and this implies that the left-hand side of (44) increases in $J_{D}$. Hence, from (41), (44) must hold when $J_{D}=J-1$, which writes as $2(1-J+N)\left(\frac{J-1}{J}\right)^{N-1}>2 N-J$. Supposing that $N>J-1$, we need

$$
\left(\frac{J-1}{J}\right)^{N-1}>\frac{2 N-J}{2-2 J+2 N}
$$

which together with $\left(\frac{J-1}{J}\right)^{N-1}<1 / 2$ would give $N<1$, which cannot be. If $N<J-1$, we need

$$
\left(\frac{J-1}{J}\right)^{N-1}<\frac{J-2 N}{2(J-1-N)}
$$

which requires $N<J / 2$ or the right-hand side would turn negative and condition (45) would be violated. Notice that both the right-hand side and the left-hand side of (45) are monotonically decreasing in $N$, so if condition (45) holds, then it must hold either when $N=2$ or when $N=J / 2$. When $N=2$, condition (45) requires $J<3$, which would violate $N<J / 2$. The condition is also violated when $N=J / 2$, showing that condition (45) is violated for any $N$. Hence, irrespective of $N$, we cannot have a profitable deviation such that $p_{D}<\tilde{p}$.

We consider the possibility of having a profitable deviation with $p_{D}=\tilde{p}$. The payoff of the deviating bank is $\min \left(J_{D} x / 2, \pi_{D}^{S}\left(J_{D}, J\right)\right)$, where

$$
\pi_{D}^{S}\left(J_{D}, J\right)=\frac{J_{D}}{J_{D}+(N-1) J}\left(1-2 \frac{J_{D}-1}{J_{D}}\left(\frac{J-1}{J}\right)^{N-1}\right) W
$$

When $J_{D}<J$, we have that $\pi_{D}^{S}\left(J_{D}, J\right)<\pi^{S}(J)$ if and only if

$$
\left(\frac{J-1}{J}\right)^{N-1}<\frac{J(N-1)}{2(1-J+J N)}
$$

Hence, full bundling is an equilibrium if (47) holds, which is the case when $N$ is sufficiently large (the left-hand side of (47) is decreasing in $N$ and the right-hand side is increasing in $N$ ). Such bound on $N$ is defined independently of $J_{D}$ since it can easily be shown that $\pi_{D}^{S}\left(J_{D}, J\right)$ increases in $J_{D}$ if and only if (47) holds, and so $\pi_{D}^{S}\left(J_{D}, J\right)<\pi^{S}(J)$ if and only if (47) holds for all $J_{D} \neq J$.

Suppose instead (47) is violated. There exists a profitable deviation if $J_{D} x / 2 \geq$ $\pi_{D}^{S}\left(J_{D}, J\right)$ or if $\pi^{S}(J)<J_{D} x / 2<\pi_{D}^{S}\left(J_{D}, J\right)$ for some $J_{D}<J$. Since when (47) is violated, we have $\pi_{D}^{S}\left(J_{D}, J\right)>\pi^{S}(J)$, it is sufficient to show that $J_{D} x / 2>\pi^{S}(J)$ and, in particular, that $(J-1) x / 2>\pi^{S}(J)$, which can be written as

$$
(J-1) \frac{x}{2} \frac{N}{W}+2\left(\frac{J-1}{J}\right)^{N}>1
$$


Hence, full bundling is an equilibrium if $N \geq \bar{N}_{S}(W)$, where

$$
\bar{N}_{S}(W)=\min _{N \in \mathbb{N}}\left\{N: \Phi_{S}(N) \leq 1\right\}
$$

and

$$
\Phi_{S}(N)=\min \left((J-1) \frac{x}{2} \frac{N}{W}+2\left(\frac{J-1}{J}\right)^{N},\left(\frac{J-1}{J}\right)^{N-1} \frac{2(1-J+J N)}{J(N-1)}\right) .
$$

Combining with (12), we have that full bundling is an equilibrium if $N \leq \hat{N}_{S}(W)$ or $N \geq$ $\bar{N}_{S}(W)$ and, provided $\bar{N}_{S}(W)>\hat{N}_{S}(W)$, it is not an equilibrium if $N \in\left(\hat{\hat{N}}_{S}(W), \bar{N}_{S}(W)\right)$.

To show that full bundling in the only equilibrium, suppose that all banks offer a bundle of size $J$ smaller than the full bundle, and that a bank deviates and offers a bundle of size $J_{D}>J$. The above analysis can be replicated exactly to show that there is no profitable deviation when $p_{D}>\tilde{p}$ or $p_{D}<\tilde{p}$. When $p_{D}=\tilde{p}$, the payoff of the deviating bank is defined by (46), and we have that $\pi_{D}^{S}\left(J_{D}, J\right)>\pi^{S}(J)$ if and only if condition (47) holds when $J_{D}>J$. Following a logic similar to that in the proof of Proposition 4, one can show that there is no other equilibrium when $N \geq \bar{N}_{S}(W)$.

Proof of Proposition 8. Preliminary results. If banks offer a pool of size $J$ and one bank deviates to a pool of size $J_{D}$, its payoff is equal to

$$
\pi_{D}\left(J_{D}, J\right)=\min \left(J_{D} x, \hat{\pi}_{D}\left(J_{D}, J\right), W / J_{D}\right)-c\left(J_{D}-J_{0}\right)^{+},
$$

with

$$
\hat{\pi}_{D}\left(J_{D}, J\right)=\frac{J_{D}}{J_{D}+(N-1) J}\left(1-\frac{J_{D}-1}{J_{D}}\left(\frac{J-1}{J}\right)^{N-1}\right) W .
$$

The payoff shown in (49) corresponds to the scenario in which investors who sample the good asset from at least one bundle buy (this is a fraction $1-\frac{J_{D}-1}{J_{D}}\left(\frac{J-1}{J}\right)^{N-1}$ of the investors), and each bundle receives a fraction of the demand in proportion to its size (so the deviating bank receives a fraction $\frac{J_{D}}{J_{D}+(N-1) J}$ of the demand). The final payoff in (48) cannot exceed the minimum between $\hat{\pi}_{D}\left(J_{D}, J\right), J_{D} x$ (since the unit price of the bundle cannot exceed $x$, otherwise no one would buy it), and $W / J_{D}$ (since the highest demand that the deviating bank can attract cannot exceed the fraction of investors who sample a good asset from its bundle).

We show that $\hat{\pi}_{D}\left(J_{D}, J\right)$ is increasing and concave in $J_{D}$. The derivative of $\hat{\pi}_{D}$ with respect to $J_{D}$ is positive if and only if

$$
\left(\frac{1}{J}(J-1)\right)^{N-1}<\frac{J(N-1)}{J(N-1)+1}
$$

When $N=2$, this requires $(J-1)(J+1)<J^{2}$, which is the case for all $J$. The condition holds a fortiori for any larger $N$. Since

$$
\frac{\partial^{2} \hat{\pi}_{D}}{\partial J_{D}^{2}}=-\frac{2}{\left(J_{D}-J+J N\right)} \frac{\partial \hat{\pi}_{D}}{\partial J_{D}},
$$

this also shows that $\hat{\pi}_{D}\left(J_{D}, J\right)$ is concave. 
We show that the payoff

$$
\Pi(J)=\min \left(\hat{\pi}_{D}(J+1, J),(J+1) x, W /(J+1)\right)-\min \left(J x,\left(1-\left(\frac{J-1}{J}\right)^{N}\right) \frac{W}{N}\right)-c
$$

cannot exceed $x-c$. First, notice that $\pi_{D}(J+1, J)-\pi(J)>x-c$ only if $\left(1-\left(\frac{J-1}{J}\right)^{N}\right) \frac{W}{N}<$ $J x$. However, if $\left(1-\left(\frac{J-1}{J}\right)^{N}\right) \frac{W}{N}<J x$, then $\hat{\pi}_{D}(J+1, J)<(J+1) x$. In fact, $\hat{\pi}_{D}(J+1, J) \geq$ $(J+1) x$ requires $\frac{J+1}{N J+1}\left(1-\frac{J+1}{J}\left(\frac{J-1}{J}\right)^{N-1}\right) \frac{W}{J+1} \geq x$, which contradicts $\left(1-\left(\frac{J-1}{J}\right)^{N}\right) \frac{W}{N J}<x$. At the same time, we have $\hat{\pi}_{D}(J+1, J)-\left(1-\left(\frac{J-1}{J}\right)^{N}\right) \frac{W}{N}<x$. In fact, $\hat{\pi}_{D}(J+1, J)-(1-$ $\left.\left(\frac{J-1}{J}\right)^{N}\right) \frac{W}{N}<\frac{N-1}{N(J N+1)}\left(1-\frac{J+1}{J}\left(\frac{J-1}{J}\right)^{N-1}\right) W<\left(1-\left(\frac{J-1}{J}\right)^{N}\right) \frac{W}{J N}<x$.

We show that $\Pi(J)$ decreases in $J$ when $\Pi(J) \geq 0$ and so $\Pi(J)$ intersects 0 only once. Notice first that if $J x<\left(1-\left(\frac{J-1}{J}\right)^{N}\right) \frac{W}{N}$, then $\Pi(J)$ is weakly decreasing in $J$. Suppose then $J x>\left(1-\left(\frac{J-1}{J}\right)^{N}\right) \frac{W}{N}$, which as shown implies $(J+1) x>\hat{\pi}_{D}(J+1, J)$. If $\hat{\pi}_{D}(J+1, J)<$ $W /(J+1)$, then

$$
\left.\Pi(J)=\hat{\pi}_{D}(J+1, J)-\left(1-\left(\frac{J-1}{J}\right)^{N}\right) \frac{W}{N}\right)-c,
$$

which is strictly decreasing in $J$ if and only if $J N-2 J+1>0$, which is the case for all $N \geq 2$ and $J$. If $\hat{\pi}_{D}(J+1, J)>W /(J+1)$, then

$$
\left.\Pi(J)=\frac{W}{J+1}-\left(1-\left(\frac{J-1}{J}\right)^{N}\right) \frac{W}{N}\right)-c,
$$

which is strictly decreasing in $J$ if and only if

$$
J+J^{2}\left(\frac{1}{J}(J-1)\right)^{N}+\left(\frac{1}{J}(J-1)\right)^{N}+2 J\left(\frac{1}{J}(J-1)\right)^{N}-J^{2}<0 .
$$

Notice that $\hat{\pi}_{D}(J+1, J)>W /(J+1)$ is written as

$$
\left(\frac{J-1}{J}\right)^{N}<\frac{N+J-J N+J^{2}-2}{J^{2}+J} .
$$

Hence, it is enough to show that (50) holds when $\left(\frac{J-1}{J}\right)^{N}=\frac{N+J-J N+J^{2}-2}{J^{2}+J}$, which requires $N+J N>3 J+2$, which is always the case for $N \geq 3$. If $N=2$, we have

$$
\left.\frac{W}{J+1}<\left(1-\left(\frac{J-1}{J}\right)^{N}\right) \frac{W}{N}\right)
$$

and so $\Pi(J)<0$ for all $c$. This shows that $\Pi(J)$ can be increasing only if $\Pi(J)<0$; hence, $\Pi(J)$ cannot intersect 0 more than once. The fact that $\Pi(J)$ must intersect 0 once follows by noticing that $\Pi(J)<0$ as $J \rightarrow \infty$.

Proof of the equilibrium. We now show that there exists an equilibrium in which all banks offer a bundle of size $\hat{J}$, with $\hat{J}=\min _{J \in \mathbb{N}}\{J: \Pi(J) \leq 0\}$. Suppose all banks offer a bundle of size $\hat{J}$ and consider a deviation with $J_{D}>\hat{J}$. Notice that by definition $\pi_{D}(\hat{J}+$ $1, \hat{J})<(\hat{J}+1) x$; hence, $\pi_{D}(\hat{J}+1, \hat{J})=\min \left(\hat{\pi}_{D}(\hat{J}+1, \hat{J}), 1 /(\hat{J}+1)\right)$. Suppose $\pi_{D}(\hat{J}+$ 
$1, \hat{J})=\hat{\pi}_{D}(\hat{J}+1, \hat{J})$. For $J_{D}>\hat{J}$, by concavity of $\hat{\pi}_{D}\left(J_{D}, \hat{J}\right),\left(\pi_{D}\left(J_{D}, \hat{J}\right)-\pi(\hat{J})\right) \leq\left(J_{D}-\right.$ $\hat{J}-1)\left(\pi_{D}(\hat{J}+1, \hat{J})-\pi(\hat{J})\right) \leq 0$, showing that there is no profitable deviation with $J_{D}>\hat{J}$. Suppose instead $\pi_{D}(\hat{J}+1, \hat{J})=1 /(\hat{J}+1)$. Then by definition of $\hat{J}, \pi_{D}(\hat{J}+1, \hat{J}) \leq \pi(\hat{J})$ and $\pi_{D}\left(J_{D}, \hat{J}\right)=1 / \hat{J}_{D}$ for all $J_{D} \geq \hat{J}+1$, showing that no deviation with $J_{D} \geq \hat{J}+1$ can be profitable.

Consider now a deviation with $J_{D}<\hat{J}$. Suppose $J_{D}=\hat{J}-1$. So as to show that $\pi_{D}(\hat{J}-$ $1, \hat{J}) \leq \pi(\hat{J})$, it is enough to show that

$$
\hat{\pi}_{D}(\hat{J}-1, \hat{J})+c \leq \min \left(\hat{J} x,\left(1-\left(\frac{\hat{J}-1}{\hat{J}}\right)^{N}\right) \frac{W}{N}\right) .
$$

Since $c<x$, (51) is always satisfied when $\left(1-\left(\frac{\hat{J}-1}{\hat{J}}\right)^{N}\right) \frac{W}{N}>\hat{J} x$. Suppose instead $\left(1-\left(\frac{\hat{J}-1}{\hat{J}}\right)^{N}\right) \frac{W}{N}<\hat{J} x$. By definition of $\hat{J}$, we have $\min \left(\hat{\pi}_{D}(\hat{J}+1, \hat{J}), 1 /(\hat{J}+1)\right)-(1-$ $\left.\left(\frac{\hat{J}-1}{\hat{J}}\right)^{N}\right) \frac{W}{N} \leq c$. Hence, (51) is satisfied if $\hat{\pi}_{D}(\hat{J}-1, \hat{J})+\min \left(\hat{\pi}_{D}(\hat{J}+1, \hat{J}), 1 /(\hat{J}+1)\right) \leq$ $2\left(1-\left(\frac{\hat{J}-1}{\hat{J}}\right)^{N}\right) \frac{W}{N}$. Since $\left(1-\left(\frac{\hat{J}-1}{\hat{J}}\right)^{N}\right) \frac{W}{N}=\hat{\pi}_{D}(\hat{J}, \hat{J})$, it is enough that

$$
\hat{\pi}_{D}(\hat{J}-1, \hat{J})+\hat{\pi}_{D}(\hat{J}+1, \hat{J}) \leq 2 \hat{\pi}_{D}(\hat{J}, \hat{J}),
$$

which is the case since $\hat{\pi}_{D}$ is concave in its first term. The concavity of $\hat{\pi}_{D}$ also implies that no $J_{D}<\hat{J}-1$ can be profitable. This shows that $\pi_{D}\left(J_{D}, \hat{J}\right)<\pi(\hat{J})$ for all $J_{D} \neq \hat{J}$ and so there is an equilibrium in which all banks offer a bundle of size $\hat{J}$.

Proof of Proposition 9. It is immediate to observe that $J^{*}$ is determined by $J_{1}$, where

$$
J_{1}: J x=\left(1-\left(\frac{J-1}{J}\right)^{N}\right) \frac{W}{N} \quad \text { at } J=J_{1} .
$$

The term $\min \left(J x,\left(1-\left(\frac{J-1}{J}\right)^{N}\right) \frac{W}{N}\right)$ in (8) increases in $J$ for $J<J_{1}$ and decreases in $J$ for $J>J_{1}$. If $J_{0} \geq J_{1}$, banks are better off by not generating any new loan. If $J_{0}<J_{1}$, the marginal benefit of an extra loan is $x$ for $J \leq J_{1}$ and the marginal cost is $c$. Due to (13), banks are better off by generating new loans up to $J_{1}$. As $J_{1}$ need not be an integer, let us define

$$
\left[J_{1}\right]=B\left(J_{1}\right),
$$

where the function $B\left(J_{1}\right)$ defines the best closest integer to $J_{1}$. That is, defining the closest integers $\left\{\breve{J}_{1}: J>J_{1}-1, J \in \mathbb{N}\right\}$ and $\left\{\bar{J}_{1}: J<J_{1}+1, J \in \mathbb{N}\right\}$, we have $B\left(J_{1}\right)=\breve{J}_{1}$ if $\breve{J}_{1} x \geq\left(1-\left(\frac{\bar{J}_{1}-1}{\bar{J}_{1}}\right)^{N}\right) \frac{W}{N}$ and $B\left(J_{1}\right)=\bar{J}_{1}$ if $\breve{J}_{1} x<\left(1-\left(\frac{\bar{J}_{1}-1}{\bar{J}_{1}}\right)^{N}\right) \frac{W}{N}$. Hence, if banks were to maximize their joint payoff, they would offer a bundle of size $J^{*}=\left[J_{1}\right]$. So as to show that there is no equilibrium with $\hat{J}<\left[J_{1}\right]$, notice that if all other banks offer $J=J_{1}$, as shown in the proof of Proposition 8, we have that $\hat{\pi}_{D}\left(J_{D}, J_{1}\right) \geq \pi\left(J_{D}\right)$ if and only if $J_{D} \geq J_{1}$ and by definition of $J_{1}, \pi\left(J_{D}\right)=J_{D} x$ when $J_{D}=J_{1}$. Hence, we have $\hat{\pi}_{D}\left(J_{D}, J_{1}\right) \geq J_{D} x$ if and only if $J_{D} \leq J_{1}$ when all other banks offer $J=J_{1}$. Since as shown in the proof of Proposition $8, \hat{\pi}_{D}\left(J_{D}, J\right)$ decreases in $J$, we have $\hat{\pi}_{D}\left(J_{D}, J_{1}\right)>J_{D} x$ for all $J_{D} \leq J_{1}$ when all other banks offer $J<J_{1}$. Since $\hat{\pi}_{D}\left(J_{D}, J_{1}\right)<1 /\left(J_{D}+1\right)$ for $J_{D} \leq J_{1}$, this implies that we 
have $\pi_{D}\left(J_{D}, J\right)=J_{D} x$ for $J_{D} \leq J_{1}$ and $J \leq J_{1}$, and so the marginal benefit of generating a new loan is $x$, which exceeds its marginal cost $c$. Hence, we cannot have $J_{D}<J_{1}$ when $J \leq J_{1}$, which implies that we cannot have a symmetric equilibrium with $\hat{J}<\left[J_{1}\right]$.

\section{REFERENCES}

Adams, William James and Janet L. Yellen (1976), "Commodity bundling and the burden of monopoly.” Quarterly Journal of Economics, 90, 475-498. [550]

Allen, Franklin and Elena Carletti (2010), "An overview of the crisis: Causes, consequences, and solutions." International Review of Finance, 10, 1-26. [548]

Allen, Franklin and Douglas Gale (1988), “Optimal security design.” Review of Financial Studies, 1, 229-263. [550]

Arora, Sanjeev, Boaz Barak, Markus Brunnermeier, and Rong Ge (2011), "Computational complexity and information asymmetry in financial products." Communications of the ACM, 54, 101-107. [549]

Baquero, Guillermo and Marno Verbeek (2008), "Do sophisticated investors believe in the law of small numbers?" Report. [549]

Barberis, Nicholas, Andrei Shleifer, and Robert Vishny (1998), "A model of investor sentiment." Journal of Financial Economics, 49, 307-343. [549]

Benartzi, Shlomo (2001), "Excessive extrapolation and the allocation of 401(k) accounts to company stock." Journal of Finance, 56, 1747-1764. [549]

Bernardo, Antonio E. and Bradford Cornell (1997), "The valuation of complex derivatives by major investment firms: Empirical evidence." Journal of Finance, 52, 785-798. [546]

Biais, Bruno and Thomas Mariotti (2005), "Strategic liquidity supply and security design.” Review of Economic Studies, 72, 615-649. [549]

Bianchi, Milo and Philippe Jehiel (2015), "Financial reporting and market efficiency with extrapolative investors." Journal of Economic Theory, 157, 842-878. [549]

Broer, Tobias (2018), "Securitization bubbles: Structured finance with disagreement about default risk.” Journal of Financial Economics, 127, 505-518. [550]

Carlin, Bruce I. (2009), "Strategic price complexity in retail financial markets." Journal of Financial Economics, 91, 278-287. [550]

Carlin, Bruce I., Francis A. Longstaff, and Kyle Matoba (2014), "Disagreement and asset prices." Journal of Financial Economics, 114, 226-238. [546]

Célérier, Claire and Boris Vallée (2017), "Catering to investors through product complexity.” Quarterly Journal of Economics, 132, 1469-1508. [548]

Chen, Joseph, Harrison Hong, and Jeremy C. Stein (2002), "Breadth of ownership and stock returns.” Journal of Financial Economics, 66, 171-205. [548] 
Coval, Joshua, Jakub Jurek, and Erik Stafford (2009), "The economics of structured finance." Journal of Economic Perspectives, 23, 3-25. [564]

Dang, Tri Vi, Gary Gorton, Bengt Holmström, and Guillermo Ordonez (2017), "Banks as secret keepers." American Economic Review, 107, 1005-1029. [549]

De Long, J. Bradford, Andrei Shleifer, Lawrence H. Summers, and Robert J. Waldmann (1990), "Positive feedback investment strategies and destabilizing rational speculation." Journal of Finance, 45, 379-395. [549]

DeMarzo, Peter (2005), "The pooling and tranching of securities: A model of informed intermediation." Review of Financial Studies, 18, 1-35. [549]

DeMarzo, Peter and Darrell Duffie (1999), "A liquidity-based model of security design." Econometrica, 67, 65-99. [549]

Diether, Karl B., Christopher J. Malloy, and Anna Scherbina (2002), "Differences of opinion and the cross section of stock returns." Journal of Finance, 57, 2113-2141. [548]

Dominitz, J. and C. F. Manski (2011), "Measuring and interpreting expectations of equity returns" Journal of Applied Econometrics, 26, 352-370. [549]

Downing, Chris, Dwight Jaffee, and Nancy Wallace (2009), "Is the market for mortgagebacked securities a market for lemons?” Review of Financial Studies, 22, 2457-2494. [548]

Ellis, Andrew, Michele Piccione, and Shengxing Zhang (2017), "Equilibrium securitization with diverse beliefs." [550]

Eyster, Erik and Michele Piccione (2013), "An approach to asset pricing under incomplete and diverse perceptions." Econometrica, 81, 1483-1506. [549]

Eyster, Erik and Matthew Rabin (2005), “Cursed equilibrium.” Econometrica, 73, 16231672. [549]

Eyster, Erik, Matthew Rabin, and Dimitri Vayanos (2019), "Financial markets where traders neglect the informational content of prices." Journal of Finance, 74, 371-399. [549]

Gabaix, Xavier and David Laibson (2006), "Shrouded attributes, consumer myopia, and information suppression in competitive markets." Quarterly Journal of Economics, 121, 505-540. [550]

Ghent, Andra C., Walter N. Torous, and Rossen I. Valkanov (2019), "Complexity in structured finance." Review of Economic Studies, 86, 694-722. [548]

Gorton, Gary and Andrew Metrick (2012), “Securitization.” [548]

Gorton, Gary and George Pennacchi (1993), "Security baskets and index-linked securities." Journal of Business, 66, 1-27. [549]

Greenwood, Robin and Stefan Nagel (2009), "Inexperienced investors and bubbles." Journal of Financial Economics, 93, 239-258. [549] 
Greenwood, Robin and Andrei Shleifer (2014), "Expectations of returns and expected returns.” Review of Financial Studies, 27, 714-746. [549]

Gul, Faruk, Wolfgang Pesendorfer, and Tomasz Strzalecki (2017), "Coarse competitive equilibrium and extreme prices." American Economic Review, 107, 109-137. [549]

Harrison, J. Michael and David M. Kreps (1978), "Speculative investor behavior in a stock market with heterogeneous expectations.” Quarterly Journal of Economics, 92, 323-336. [549]

Henderson, Brian J. and Neil D. Pearson (2011), "The dark side of financial innovation: A case study of the pricing of a retail financial product." Journal of Financial Economics, 100, 227-247. [548]

Jehiel, Philippe (2005), “Analogy-based expectation equilibrium.” Journal of Economic Theory, 123, 81-104. [549]

Jehiel, Philippe and Frédéric Koessler (2008), "Revisiting games of incomplete information with analogy-based expectations." Games and Economic Behavior, 62, 533-557. [549]

Jehiel, Philippe, Moritz Meyer-Ter-Vehn, and Benny Moldovanu (2007), "Mixed bundling auctions." Journal of Economic Theory, 134, 494-512. [550]

Kondor, Péter and Botond Koszegi (2017), "Financial choice and financial information.” [549]

Krugman, Paul (2007), “Innovating our way to financial crisis.” [546]

Maddaloni, Angela and José-Luis Peydró (2011), "Bank risk-taking, securitization, supervision, and low interest rates: Evidence from the Euro-area and the US lending standards." Review of Financial Studies, 24, 2121-2165. [548]

McAfee, R. Preston, John McMillan, and Michael D. Whinston (1989), "Multiproduct monopoly, commodity bundling, and correlation of values.” Quarterly Journal of Economics, 104, 371-383. [550]

Miller, Edward M. (1977), “Risk, uncertainty, and divergence of opinion.” Journal of finance, 32, 1151-1168. [549]

Myers, Stewart and Nicholas Majluf (1984), "Corporate financing and investment decisions when firms have information that investors do not have." Journal of Financial Economics, 13, 187-221. [549]

Osborne, Martin J. and Ariel Rubinstein (1998), "Games with procedurally rational players.” American Economic Review, 88, 834-847. [549]

Palfrey, Thomas R. (1983), "Bundling decisions by a multiproduct monopolist with incomplete information.” Econometrica, 51, 463-483. [550]

Purnanandam, Amiyatosh (2010), "Originate-to-distribute model and the subprime mortgage crisis.” Review of Financial Studies, 24, 1881-1915. [548] 
Rabin, Matthew (2002), "Inference by believers in the law of small numbers." Quarterly Journal of Economics, 117, 775-816. [549]

Rabin, Matthew and Dimitri Vayanos (2010), “The gambler's and hot-hand fallacies: Theory and applications." Review of Economic Studies, 77, 730-778. [549]

Scheinkman, Jose A. and Wei Xiong (2003), "Overconfidence and speculative bubbles.” Journal of Political Economy, 111, 1183-1219. [549]

Shiller, Robert J. (2000), “Measuring bubble expectations and investor confidence.” Journal of Psychology and Financial Markets, 1, 49-60. [549]

Simsek, Alp (2013), "Speculation and risk sharing with new financial assets." Quarterly Journal of Economics, 128, 1365-1396. [549]

Skreta, Vasiliki and Laura Veldkamp (2009), "Ratings shopping and asset complexity: A theory of ratings inflation." Journal of Monetary Economics, 56, 678-695. [546]

Soros, George (2009), The Crash of 2008 and What It Means: The New Paradigm for Financial Markets. PublicAffairs. [546]

Spiegler, Ran (2006), “The market for quacks.” Review of Economic Studies, 73, 11131131. [549]

Spiegler, Ran (2016), “Competition and obfuscation.” Annual Review of Economics, 8. [550]

Steiner, Jakub and Colin Stewart (2015), "Price distortions under coarse reasoning with frequent trade.” Journal of Economic Theory, 159, 574-595. [549]

Tversky, Amos and Daniel Kahneman (1971), "Belief in the law of small numbers." Psychological Bulletin, 76, 105-110. [549]

Tversky, Amos and Daniel Kahneman (1975), "Judgment under uncertainty: Heuristics and biases." In Utility, Probability, and Human Decision Making, 141-162, Springer. [549]

Xiong, W. (2013), "Bubbles, crises, and heterogeneous beliefs." In Handbook on Systemic Risk, 663-713, Cambridge University Press. [549]

Co-editor Florian Scheuer handled this manuscript.

Manuscript received 16 April, 2019; final version accepted 1 October, 2019; available online 10 October, 2019. 